\title{
Relativistic supernova 2009bb exploded close to an atomic gas cloud ${ }^{\star}$
}

\author{
M. J. Michałowski ${ }^{1,2}$, G. Gentile ${ }^{3,4}$, T. Krühler ${ }^{5,6}$, H. Kuncarayakti ${ }^{7,8}$, P. Kamphuis ${ }^{9}$, J. Hjorth ${ }^{10}$, \\ S. Berta ${ }^{11}$, V. D'Elia ${ }^{12,13}$, J. Elliott ${ }^{6,14}$, L. Galbany ${ }^{15}$, J. Greiner ${ }^{6}$, L. K. Hunt ${ }^{16}$, M. P. Koprowski ${ }^{17}$, \\ E. Le Floc'h ${ }^{18}$, A. Nicuesa Guelbenzu ${ }^{19}$, E. Palazzi ${ }^{20}$, J. Rasmussen ${ }^{10,21}$, A. Rossi $^{20}$, S. Savaglio $^{22}$, \\ A. de Ugarte Postigo ${ }^{23,10}$, P. van der Werf ${ }^{24}$, and S. D. Vergani ${ }^{25}$
}

(Affiliations can be found after the references)

Received 24 November 2017 / Accepted 12 July 2018

\begin{abstract}
Context. The host galaxies of gamma-ray bursts (GRBs) have been claimed to have experienced a recent inflow of gas from the intergalactic medium. This is because their atomic gas distribution is not centred on their optical emission and because they are deficient in molecular gas given their high star formation rates (SFRs). Similar studies have not been conducted for host galaxies of relativistic supernovae (SNe), which may have similar progenitors.

Aims. The potential similarity of the powering mechanisms of relativistic SNe and GRBs allowed us to make a prediction that relativistic SNe are born in environments similar to those of GRBs, that is, ones which are rich in atomic gas. Here we embark on testing this hypothesis by analysing the properties of the host galaxy NGC 3278 of the relativistic SN 2009bb. This is the first time the atomic gas properties of a relativistic SN host are provided and the first time resolved $21 \mathrm{~cm}$-hydrogen-line (H I) information is provided for a host of an SN of any type in the context of the SN position.

Methods. We obtained radio observations with the Australia Telescope Compact Array (ATCA) covering the H I line, and optical integral field unit spectroscopy observations with the Multi Unit Spectroscopic Explorer (MUSE) at the Very Large Telescope (VLT). Moreover, we analysed archival carbon monoxide (CO) and multi-wavelength data for this galaxy.

Results. The atomic gas distribution of NGC 3278 is not centred on the optical galaxy centre, but instead around a third of atomic gas resides in the region close to the SN position. This galaxy has a few times lower atomic and molecular gas masses than predicted from its SFR. Its specific SFR ( $\mathrm{SSFR} \equiv \mathrm{SFR} / M_{*}$ ) is approximately two to three times higher than the main-sequence value, placing it at the higher end of the main sequence, towards starburst galaxies. SN 2009bb exploded close to the region with the highest SFR density and the lowest age, as evident from high H $\alpha$ EW, corresponding to the age of the stellar population of $\sim 5.5 \mathrm{Myr}$. Assuming this timescale was the lifetime of the progenitor star, its initial mass would have been close to $\sim 36 M_{\odot}$.

Conclusions. As for GRB hosts, the gas properties of NGC 3278 are consistent with a recent inflow of gas from the intergalactic medium, which explains the concentration of atomic gas close to the SN position and the enhanced SFR. Super-solar metallicity at the position of the SN (unlike for most GRBs) may mean that relativistic explosions signal a recent inflow of gas (and subsequent star formation), and their type (GRBs or SNe) is determined either (i) by the metallicity of the inflowing gas, so that metal-poor gas results in a GRB explosion and metal-rich gas (for example a minor merger with an evolved galaxy or re-accretion of expelled gas) results in a relativistic SN explosion without an accompanying GRB, or (ii) by the efficiency of gas mixing (efficient mixing for SN hosts leading to a quick disappearance of metal-poor regions), or (iii) by the type of the galaxy (more metal-rich galaxies would result in only a small fraction of star formation being fuelled by metal-poor gas).
\end{abstract}

Key words. dust, extinction - galaxies: individual: NGC 3278 - galaxies: ISM - galaxies: star formation - supernovae: individual: SN 009bb radio lines: galaxies

\section{Introduction}

The gas inflow from the intergalactic medium is predicted to be an important process providing the fuel for star formation (see e.g. Sancisi et al. 2008; Spring \& Michałowski 2017). It has been studied mostly from indirect diagnostics because compiling a sample of galaxies for which this process can be observed directly is difficult.

Based on the analysis of gas properties in long gammaray burst (GRB) host galaxies, we have recently proposed that the progenitors of GRBs are preferentially born when a galaxy accretes fresh gas from the intergalactic medium (Michałowski et al. 2015, 2016). This is based on a high

\footnotetext{
* The reduced VLA and MUSE datacubes (FITS files) are only available at the CDS via anonymous ftp to cdsarc.u-strasbg.fr (130.79.128.5) or via http://cdsarc.u-strasbg.fr/viz-bin/ qcat?J $/ \mathrm{A}+\mathrm{A} / 618 / \mathrm{A} 104$
}

abundance of atomic gas in GRB hosts and its concentration close to the GRB position (Arabsalmani et al. 2015; Michałowski et al. 2015). This may also imply that a fraction of star formation is fuelled directly by atomic, not molecular, gas. The majority of star formation in the Universe is fuelled by molecular gas, as shown by many observations (e.g. Carilli \& Walter 2013; Rafelski et al. 2016). However, H I-fuelled star formation has been shown to be theoretically possible (Glover \& Clark 2012; Krumholz 2012; Hu et al. 2016) and it was supported by the existence of $\mathrm{HI}$-dominated, star-forming regions in other galaxies (Bigiel et al. 2008, 2010; Fumagalli \& Gavazzi 2008; Elmegreen et al. 2016). If the connection between GRBs and recent inflow is confirmed, this will allow the use of GRB hosts to study gas accretion and/or H I-fuelled star formation.

On the other hand, relativistic supernovae ( $\mathrm{SNe}$ ) without detected $\gamma$-rays are thought to be powered by similar engines to those of GRBs, but with the jet failing to break out 
from the exploding star (Paragi et al. 2010; Lazzati et al. 2012; Margutti et al. 2014; Chakraborti et al. 2015; Milisavljevic et al. 2015). The potential similarity of this powering mechanism to that of GRBs allowed us to make a prediction that relativistic $\mathrm{SNe}$ are born in environments similar to those of GRBs, that is, those rich in atomic gas. Here we embark on testing this hypothesis by analysing the properties of the host of the relativistic SN 2009bb.

SN 2009bb was discovered by the galaxy-targeted survey, the CHilean Automatic Supernova sEarch (CHASE; Pignata et al. 2009a) on 21 March 2009 (Pignata et al. 2009b) at the position of 10:31:33.8762,-39:57:30.022 (Bietenholz et al. 2010) and was a broad-line type-Ic supernova (Stritzinger et al. 2009). Radio and optical behaviour, and the relativistic ejecta velocity of SN 2009 bb were very similar to those of low $-z$ GRBs, especially GRB 980425 (SN1998bw; Soderberg et al. 2010; Bietenholz et al. 2010; Pignata et al. 2011; De Colle et al. 2018).

SN 2009bb exploded within a spiral galaxy type Sa (de Vaucouleurs et al. 1991) NGC 3278 (ESO 317-G 043, PGC 031068 ) at a redshift of $0.009877 \pm 0.000123$ (Strauss et al. 1992). It has an inclination to the line of sight of $41 \mathrm{deg}$ (Makarov et al. 2014) ${ }^{1}$. The SN 2009bb explosion site was reported to have super-solar metallicity (Levesque et al. 2010c).

The objectives of this paper are: (i) to provide the first resolved measurement of the atomic gas properties of a relativistic SN host, (ii) to test whether these properties are consistent with a recent inflow of atomic gas from the intergalactic medium, and (iii) to derive the properties of NGC 3278 to assess the possible implications regarding the nature of the progenitor of SN 2009bb.

We use a cosmological model with $H_{0}=70 \mathrm{~km} \mathrm{~s}^{-1} \mathrm{Mpc}^{-1}$, $\Omega_{\Lambda}=0.7$, and $\Omega_{m}=0.3$, so SN 2009bb at $z=0.009877$ is at a luminosity distance of $42.6 \mathrm{Mpc}$ and $1^{\prime \prime}$ corresponds to $203 \mathrm{pc}$ at its redshift. We also assume the Chabrier (2003) initial mass function (IMF), to which all star formation rate (SFR) and stellar masses were converted (by dividing by 1.8 ) if given originally assuming the Salpeter (1955) IMF.

\section{Data}

\subsection{Radio}

We performed radio observations with the Australia Telescope Compact Array (ATCA) using the Compact Array Broad-band Backend (CABB; Wilson et al. 2011) on 8 March 2016 (project no. C2700, PI: M. Michałowski). The array was in the $6 \mathrm{~B}$ configuration with baselines $214-5939 \mathrm{~m}$. The total integration time was $\sim 6.5 \mathrm{~h}$. Sources 1934-638 and 1018-426 were used as the primary and secondary calibrator, respectively. The data reduction and analysis were done using the MIRIAD package (Sault \& Killeen 2004; Sault et al. 1995).

An intermediate frequency (IF) was centred at the Hi line in the ATCA CABB "zoom" mode with $32 \mathrm{kHz}$ resolution. We subtracted the continuum to obtain the continuum-free data, and made the Fourier inversion with the Brigg's weighting robust parameter of 0.5 , inverting five channels at a time to get a data cube with a velocity resolution of $33 \mathrm{~km} \mathrm{~s}^{-1}$. We then made a CLEAN deconvolution down to $\sim 3 \sigma$, after which we restored the channel maps with a Gaussian beam with the size of $36 \times 28^{\prime \prime}$ and a position angle of $19 \mathrm{deg}$ (from the north towards east). We obtained the rms of $\sim 1.5 \mathrm{mJy}$ at $33 \mathrm{~km} \mathrm{~s}^{-1}$ channels. We used a $80^{\prime \prime}$ diameter aperture to measure the fluxes for the entire host, and $30^{\prime \prime}$ for the H I peak (see Fig. A.1).

1 http://leda.univ-lyon 1 . fr/ledacat. cgi?NGC\%203278

\section{2. $\mathrm{CO}$}

We used the $\mathrm{CO}(1-0)$ and $\mathrm{CO}(2-1)$ data obtained with the Swedish European Southern Observatory (ESO) Submillimeter Telescope (SEST) by Albrecht et al. (2007). The beam sizes are 45 and $24^{\prime \prime}$, respectively. We estimated the molecular gas mass from the $\mathrm{CO}(1-0)$ line luminosity assuming the Galactic CO-to$\mathrm{H}_{2}$ conversion factor $\alpha_{\mathrm{CO}}=5 M_{\odot} /\left(\mathrm{K} \mathrm{km} \mathrm{s}^{-1} \mathrm{pc}^{2}\right)$. For completeness we also estimated the molecular mass from the $\mathrm{CO}(2-1)$ line using the flux conversion $S_{\mathrm{CO}(1-0)}=0.5 \times S_{\mathrm{CO}(2-1)}$ (Fig. 4 in Carilli \& Walter 2013), $L_{\mathrm{CO}(1-0)}^{\prime}=2 \times L_{\mathrm{CO}(2-1)}^{\prime}$. However, as we possess the $\mathrm{CO}(1-0)$ measurement, we do not use the mass based on $\mathrm{CO}(2-1)$ in the analysis.

\subsection{Optical integral field spectroscopy}

We obtained the observations of NGC 3278 using the Multi Unit Spectroscopic Explorer (MUSE; Bacon et al. 2010) at the Very Large Telescope (VLT) on 15 May 2015 (proposal 095.D0172(A), PI: H. Kuncarayakti, see Kuncarayakti et al. 2018 for other results from this programme). The data acquisition and reduction was similar to that described in Krühler et al. (2017). The total integration time was $0.5 \mathrm{~h}$. The seeing was around $1^{\prime \prime}$. The data covers a region of $60^{\prime \prime} \times 60^{\prime \prime}$ and the wavelength range $0.475-0.93 \mu \mathrm{m}$. To reduce the data, we used the ESO MUSE pipeline ${ }^{2}$ (Weilbacher et al. 2014) in the standard manner. The datacube was corrected for the Milky Way extinction $E_{B-V}=0.085$ mag (Schlafly \& Finkbeiner 2011).

As in Galbany et al. (2014), in order to obtain the galactocentric distance of each pixel, we used the code developed by Krajnović et al. (2006). It analyses the velocity field of the galaxy to obtain the position angle ( $156 \mathrm{deg}$ for NGC 3278) and the axes ratio $(\sim 0.64)$. We also derived the inclination to the line of sight of $\sim 50 \mathrm{deg}$, close to the value of $41 \mathrm{deg}$ given by Makarov et al. (2014). In this way the maps of the deprojected distances were obtained and used for radial dependence of estimated properties.

\subsection{Broad-band photometry}

We used the photometry for NGC 3278 listed in the NASA/IPAC Extragalactic Database (NED). This includes optical $(B, R$; Lauberts \& Valentijn 1989), near-infrared ( $, H, K$; Jarrett et al. 2000; Skrutskie et al. 2006), mid- and far-infrared (12, 25, 60, $100 \mu \mathrm{m}$; Sanders et al. 2003), and radio $(1.4,0.843 \mathrm{GHz}$; Condon et al. 1998; Mauch et al. 2003) data. Additionally we used the $617 \mathrm{MHz}$ flux reported in Soderberg et al. (2010).

We also used the data from the Wide-field Infrared Survey Explorer (WISE; Wright et al. 2010). We used the fluxes from the AllWISE Source Catalog ${ }^{3}$ measured in elliptical apertures with semi-major axes of 31.60-33.83" (w [F] gmag, F $\in$ $1,2,3,4)$, which we list in Table 1.

Finally, we used the VLA $1.4 \mathrm{GHz}$ continuum data from Condon et al. (1996). The image has $18^{\prime \prime}$ resolution allowing us to investigate the spatial distribution of star formation.

\section{SED modelling}

For the host galaxy emission, we applied the spectral energy distribution (SED) fitting method detailed in Michałowski et al. (2008, 2009, 2010a,b, 2012, 2014a, see therein a discussion

\footnotetext{
www.eso.org/sci/software/pipelines/

http://irsa.ipac.caltech.edu/Missions/wise.html
} 
Table 1. WISE fluxes of NGC 3278.

\begin{tabular}{ccc}
\hline \hline$\lambda / \mu \mathrm{m}$ & Flux $/ \mathrm{mJy}$ & aperture/arcsec \\
\hline 3.4 & $68.47 \pm 0.38$ & 31.60 \\
4.6 & $45.98 \pm 0.25$ & 31.60 \\
12 & $327.7 \pm 1.8$ & 31.60 \\
22 & $524.8 \pm 3.4$ & 33.83 \\
\hline
\end{tabular}

of the derivation of galaxy properties and typical uncertainties), which is based on 35000 templates from the library of Iglesias-Páramo et al. (2007) plus some templates from Silva et al. (1998) and Michałowski et al. (2008), all of which were developed using GRASIL $^{4}$ (Silva et al. 1998). They are based on numerical calculations of radiative transfer within a galaxy, which is assumed to be a triaxial axisymmetric system with diffuse dust and dense molecular clouds in which stars are born.

The templates cover a broad range of galaxy properties from quiescent to starburst, and span an $A_{\mathrm{V}}$ range from 0 to $5.5 \mathrm{mag}$. The extinction curve (Fig. 3 of Silva et al. 1998) is derived from the modified dust grain size distribution of Draine \& Lee (1984). The star formation histories are assumed to be a smooth Schmidt-type law (i.e. the SFR is proportional to the gas mass; see Silva et al. 1998, for details) with a starburst (if any) on top of that, starting $50 \mathrm{Myr}$ before the time at which the SED is computed. There are seven free parameters in the library of Iglesias-Páramo et al. (2007): the normalisation of the Schmidttype law, the timescale of the mass infall, the intensity of the starburst, the timescale for molecular cloud destruction, the optical depth of the molecular clouds, the age of the galaxy, and the inclination of the disk with respect to the observer.

We also used MAGPHYS ${ }^{5}$ (Multi-wavelength Analysis of Galaxy Physical Properties; da Cunha et al. 2008), which is an empirical, physically-motived SED modelling code that is based on the energy balance between the energy absorbed by dust and that re-emitted in the infrared. We used the Bruzual \& Charlot (2003) stellar population models and adopted the Chabrier (2003) IMF.

Similarly to GRASIL, in MAGPHYS two dust media are assumed: a diffuse interstellar medium (ISM) and dense stellar birth clouds. Four dust components are taken into account: cold dust (15-25 K), warm dust (30-60 K), hot dust (130-250 K), and polycyclic aromatic hydrocarbons (PAHs). A simple power-law attenuation law is assumed.

\section{Results}

\subsection{Integrated stellar properties}

The best-fit SED models are presented in Fig. 1 compared with the host galaxies of GRB 980425 (Michałowski et al. 2014b) and 111005A (Michałowski et al. 2018b). The derived galaxy properties are listed in Tables 2 and 3. Our derived SFR is consistent with that reported in Levesque et al. (2010c).

In terms of stellar mass, NGC 3278 is a typical galaxy with $M_{*} \sim 3 \times 10^{10} M_{\odot}$, close to the knee of the stellar mass function of local spiral galaxies (Moffett et al. 2016). However, the specific SFR ( $\mathrm{SSFR} \equiv \mathrm{SFR} / M_{*}$ ) of $0.08-0.14 \mathrm{Gyr}^{-1}$ is approximately two to three times higher than the main-sequence value of $0.04-0.05 \mathrm{Gyr}^{-1}$ at this redshift and mass (Speagle et al. 2014).

\footnotetext{
4 adlibitum.oats.inaf.it/silva/grasil/grasil.html
} www.iap.fr/magphys

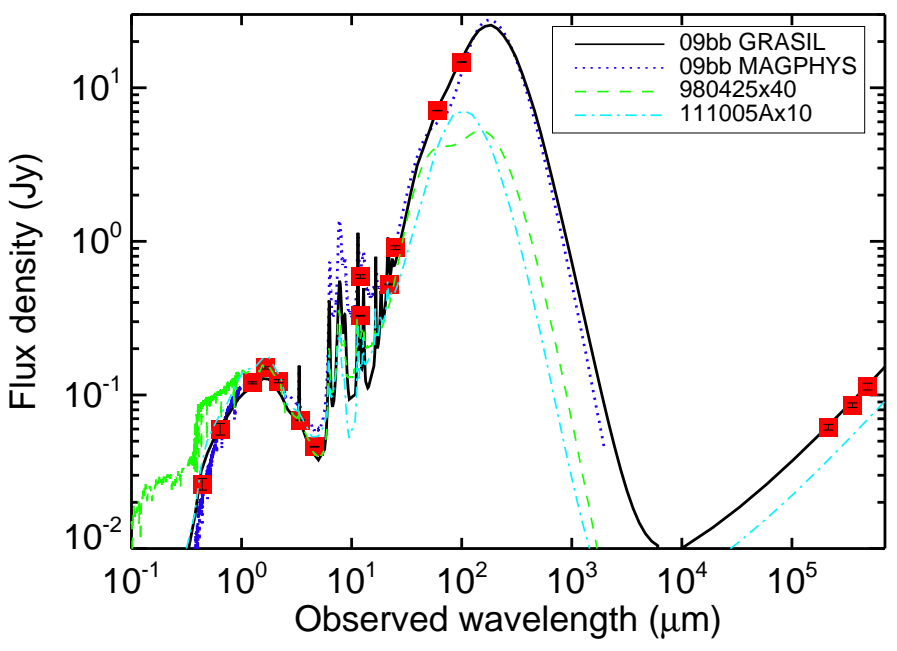

Fig. 1. Spectral energy distribution of the NGC 3278 (red points). The Grasil and MAGPHYs models are shown as black solid and blue dotted lines, respectively. For comparison we show the models for the hosts of GRB 980425 (Michałowski et al. 2014b) and 111005A (Michałowski et al. 2018b), scaled to approximately match near-IR fluxes.

Hence, NGC 3278 is at the higher end of the main sequence towards starburst galaxies. The stellar mass of NGC 3278 is at least an order of magnitude higher than those of low- $z$ GRB hosts (Savaglio et al. 2009; Castro Cerón et al. 2010; Vergani et al. 2015; Japelj et al. 2016; Perley et al. 2016b, but see an atypical massive and quiescent host presented by Rossi et al. 2014).

Both models return quite high levels of visual dust attenuation $\left(A_{\mathrm{V}} \sim 1-2 \mathrm{mag}\right)$, which is evidenced by red optical colours, similar to those of the GRB 111005A host; however, that galaxy is nearly edge-on, whereas NGC 3278 has an inclination of $41 \mathrm{deg}$, so the amount of dust is much higher. Indeed the dust mass of NGC 3278 is approximately two orders of magnitude higher than that of the GRB 111005A host (whereas the stellar mass of the latter is less than a factor of ten smaller). The dust mass estimates of NGC 3278 with GrasIL and MAGPHYS differ by an order of magnitude, but the lack of long-wavelength data above $100 \mu \mathrm{m}$ means that this parameter is very poorly constrained. A factor of two difference is due to different mass absorption coefficients $\kappa$ and the rest is due to differences in assumed temperatures and distributions of dust components.

\subsection{Gas properties}

The Hi fluxes at each frequency element were determined by aperture photometry with the aperture radius of $80^{\prime \prime}$ for the entire galaxy and of $30^{\prime \prime}$ for the H I peak. When we, instead, fit a two-dimensional (2D) Gaussian with the size of the beam at the position of the HI peak, we obtained the Hi flux of $\sim 1.30 \pm 0.17 \mathrm{Jy} \mathrm{km} \mathrm{s}^{-1}$, consistent with the aperture estimate of $\sim 1.53 \pm 0.17 \mathrm{Jy} \mathrm{km} \mathrm{s}^{-1}$

The spectra are shown in Fig. 2. Gaussian functions were fitted to them and the parameters of the fit are reported in Cols. 2-4 of Table 4. The H I emission map derived from the collapsed cube within $2 \sigma$ from this fit (dotted lines in Fig. 2) is shown in Fig. 3. This range was also used to obtain integrated $\mathrm{H} \mathrm{I}$ emission $\left(F_{\text {int }}\right.$ in $\left.\mathrm{Jy} \mathrm{km} \mathrm{s}{ }^{-1}\right)$ directly from the spectra (not from the Gaussian fit, which is not a perfect representation of the line shape). The line luminosity $\left(L_{\mathrm{HI}}^{\prime}\right.$ in $\left.\mathrm{K} \mathrm{km} \mathrm{s}^{-1} \mathrm{pc}^{2}\right)$ was calculated using Eq. (3) in Solomon et al. (1997) and transformed to $M_{\mathrm{HI}}$ 
Table 2. MaGPHYs results from the SED fitting.

\begin{tabular}{|c|c|c|c|c|c|c|c|c|c|c|c|c|c|}
\hline $\begin{array}{l}\log L_{\mathrm{IR}} \\
\left(L_{\odot}\right) \\
(1)\end{array}$ & $\begin{array}{c}\text { SFR } \\
\left(M_{\odot} \mathrm{yr}^{-1}\right) \\
(2) \\
\end{array}$ & $\begin{array}{c}\text { sSFR } \\
\left(\mathrm{Gyr}^{-1}\right) \\
(3)\end{array}$ & $\begin{array}{c}\log M_{*} \\
\left(M_{\odot}\right) \\
(4)\end{array}$ & $\begin{array}{c}\log M_{d} \\
\left(M_{\odot}\right) \\
(5)\end{array}$ & $\begin{array}{l}\tau_{\mathrm{V}} \\
(6) \\
\end{array}$ & $\begin{array}{l}T_{\text {cold }} \\
(\mathrm{K}) \\
(7) \\
\end{array}$ & $\begin{array}{c}\xi_{\text {cold }} \\
(8) \\
\end{array}$ & $\begin{array}{c}T_{\text {warm }} \\
(\mathrm{K}) \\
(9)\end{array}$ & $\begin{array}{c}\xi_{\text {warm }} \\
(10)\end{array}$ & $\begin{array}{l}\xi_{\text {hot }} \\
(11)\end{array}$ & $\begin{array}{l}\xi_{\mathrm{PAH}} \\
(12) \\
\end{array}$ & $\begin{array}{c}f_{\mu} \\
(13) \\
\end{array}$ & $\begin{array}{c}\log \operatorname{age}_{M} \\
(\mathrm{yr}) \\
(14)\end{array}$ \\
\hline $10.72_{-0.03}^{+0.06}$ & $2.77_{-0.85}^{+0.88}$ & $0.08_{-0.03}^{+0.07}$ & $10.54_{-0.15}^{+0.12}$ & $7.97_{-0.44}^{+0.48}$ & $2.42_{-0.84}^{+1.23}$ & $18.9_{-2.5}^{+3.2}$ & $0.43_{-0.03}^{+0.04}$ & $55_{-3}^{+4}$ & $0.30_{-0.03}^{+0.04}$ & $0.11_{-0.02}^{+0.02}$ & $0.154_{-0.022}^{+0.021}$ & $0.66_{-0.08}^{+0.08}$ & $9.84_{-0.10}^{+0.10}$ \\
\hline
\end{tabular}

Notes. (1) 8-1000 $\mu \mathrm{m}$ infrared luminosity. (2) Star formation rate from SED modelling. (3) Specific star formation rate (三 SFR/M*). (4) Stellar mass. (5) Dust mass. (6) Average $V$-band optical depth $\left(A_{\mathrm{V}}=1.086 \tau_{\mathrm{V}}\right)$. (7) Temperature of the cold dust component. (8) Contribution of the cold component to the infrared luminosity. (9) Temperature of the warm dust component. (10) Contribution of the warm component to the infrared luminosity. (11) Contribution of the hot (130-250 K, mid-IR continuum) component to the infrared luminosity. (12) Contribution of the PAH component to the infrared luminosity. (13) Contribution of the ISM dust (as opposed to birth clouds) to the infrared luminosity. (14) Mass-weighted age.

Table 3. Grasil results from the SED fitting.

\begin{tabular}{|c|c|c|c|c|c|c|c|c|c|}
\hline $\begin{array}{c}\log L_{\mathrm{IR}} \\
\left(L_{\odot}\right) \\
(1) \\
\end{array}$ & $\begin{array}{c}\mathrm{SFR}_{\mathrm{IR}} \\
\left(M_{\odot} \mathrm{yr}^{-1}\right) \\
(2) \\
\end{array}$ & $\begin{array}{c}\mathrm{SFR}_{\mathrm{SED}} \\
\left(M_{\odot} \mathrm{yr}^{-1}\right) \\
(3)\end{array}$ & $\begin{array}{c}\mathrm{SFR}_{\mathrm{UV}} \\
\left(M_{\odot} \mathrm{yr}^{-1}\right) \\
(4) \\
\end{array}$ & $\begin{array}{c}\text { sSFR } \\
\left(\mathrm{Gyr}^{-1}\right) \\
(5)\end{array}$ & $\begin{array}{c}\log M_{*} \\
\left(M_{\odot}\right) \\
(6) \\
\end{array}$ & $\begin{array}{c}\log M_{\text {dust }} \\
\left(M_{\odot}\right) \\
(7) \\
\end{array}$ & $\begin{array}{c}\log T_{\text {dust }} \\
(\mathrm{K}) \\
(8) \\
\end{array}$ & $\begin{array}{c}A_{\mathrm{V}} \\
(\mathrm{mag}) \\
(9)\end{array}$ & $\begin{array}{l}\log \operatorname{age}_{M} \\
\text { (yr) } \\
\text { (10) } \\
\end{array}$ \\
\hline 10.74 & 5.21 & 3.69 & 0.54 & 0.14 & 10.43 & 8.93 & 21 & 0.96 & 9.93 \\
\hline
\end{tabular}

Notes. (1) 8-1000 $\mu \mathrm{m}$ infrared luminosity. (2) Star formation rate from $L_{\mathrm{IR}}$ (Kennicutt 1998). (3) Star formation rate from SED modelling. (4) Star formation rate from UV emission (Kennicutt 1998). (5) Specific star formation rate (三SFR $\mathrm{SED}_{\text {/ }} M_{*}$ ). (6) Stellar mass. (7) Dust mass. (8) Dust temperature. (9) Mean dust attenuation at $V$-band. (10) Mass-weighted age.

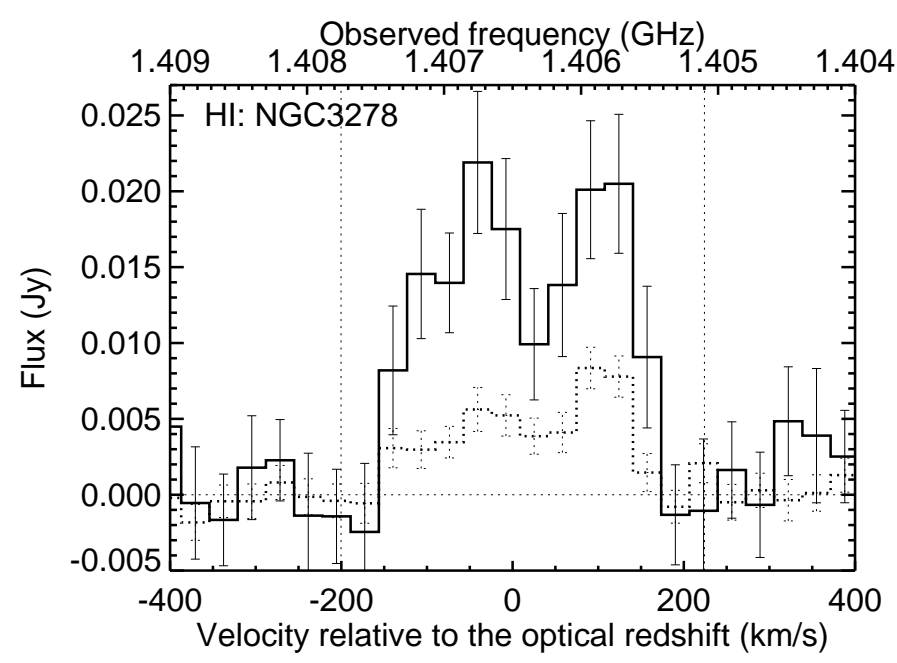

Fig. 2. H I spectrum of NGC 3278 extracted over the entire galaxy within an aperture of $80^{\prime \prime}$ radius (solid histogram) and of the dominant H I region (see Fig. 3) within an aperture of 30" radius (dotted histogram).

using Eq. (2) in Devereux \& Young (1990). For the HI peak the flux corresponds to the neutral hydrogen column density of $\sim(1.67 \pm 0.19) \times 10^{21} \mathrm{~cm}^{2}$.

The Hi first moment map (velocity field) is shown on the right panel of Fig. 3. The large beam does not allow detailed velocity analysis, but the field does not resemble clearly a rotating disk (positive velocities are on both sides of regions with negative velocities). On the other hand, the $\mathrm{H}$ i spectrum exhibits a double-peaked profile characteristic of a rotating disk, but the significance of this feature is low. Therefore it is likely that only a fraction of atomic gas is within a rotating disk giving rise to this double-peaked profile.

We detected and resolved the $\mathrm{HI}$ emission of the target, so that we are able to identify the main concentration of atomic gas. This is the first time H I data for a relativistic SN host is provided and the first time resolved $\mathrm{H}_{\mathrm{I}}$ information is analysed for the host of an SN of any type in the context of the SN position (non-resolved results were presented in Galbany et al. 2017).

The emission is not concentrated near the galaxy centre, but towards the SN position (the peak is $\sim 21^{\prime \prime}$ [ $\left.\sim 4 \mathrm{kpc}\right]$, i.e. one beam, south of the $\mathrm{SN}$ position). This concentration is responsible for $\sim 32 \pm 5 \%$ of the total integrated flux. The remaining emission probably comes from a rotating disk (giving rise to the double-peaked H I profile in Fig. 2). It seems that the sensitivity of our data allowed us to clearly detect only the strongest concentration of $\mathrm{HI}$, leaving the emission from the disk difficult to identify.

Given limited $u v$-coverage, we investigated the issue of whether we resolve out a significant fraction of the $\mathrm{H}$ i flux. Our observations in the ATCA 6B configuration are limited by a largest recoverable scale of $\sim 105^{\prime \prime}$ (Table 1.5 in the ATCA Users Guide $^{6}$, a more optimistic estimate based on the ratio of the observed wavelength and the shortest baseline of $214 \mathrm{~m}$ gives $\sim 200^{\prime \prime}$ ). This is larger than the optical extent of the galaxy (diameter of $\sim 60^{\prime \prime} \times 40^{\prime \prime}$ ), so our observations are unlikely to resolve out a lot of $\mathrm{H}$ i emission. Even if the atomic gas disk is a few times larger than the optical disk (which is not uncommon) and we do resolve out some of the extended emission, then our conclusion is still valid that the strongest atomic gas concentration is located away from the galaxy centre towards the SN position. A similar strong H I concentration away from the galaxy centre was detected for the GRB 980425 host by the Giant Metrewave Radio Telescope (GMRT; Arabsalmani et al. 2015).

However, it is unlikely that we resolve out a significant fraction of the total emission, because our measurement agrees with the single-dish flux. Courtois et al. (2011) and Roth et al. (1994) provided low resolution Green Bank Telescope and Parkes H I data for NGC 3278 (as a part of a larger survey of local galaxies, so they did not discuss that this was a relativistic SN host).

6 wwW.narrabri.atnf.csiro.au/observing/users_guide/ html/atug.html 
Table 4. H I properties of NGC 3278.

\begin{tabular}{lccccccc}
\hline \hline Region & $z_{\mathrm{HI}}$ & $\begin{array}{c}v_{\mathrm{HI}} \\
\left(\mathrm{km} \mathrm{s}^{-1}\right) \\
(3)\end{array}$ & $\begin{array}{c}v_{\mathrm{FWHM}} \\
\left(\mathrm{km} \mathrm{s}^{-1}\right)\end{array}$ & $\begin{array}{c}F_{\text {peak }} \\
(4)\end{array}$ & $\begin{array}{c}F_{\text {int }} \\
(5)\end{array}$ & $\begin{array}{c}\log \left(L_{\mathrm{HI}}^{\prime}\right) \\
(6)\end{array}$ & $\begin{array}{c}\log \left(M_{\mathrm{HI}}\right) \\
\left(\mathrm{Jm} \mathrm{km} \mathrm{s}_{\odot}\right) \\
(7)\end{array}$ \\
\hline NGC 3278 & $0.009890 \pm 0.000040$ & $2965 \pm 12$ & $222 \pm 27$ & $19.2 \pm 1.9$ & $4.84 \pm 0.53$ & $11.148 \pm 0.045$ & $9.318 \pm 0.045$ \\
H I peak & $0.009983 \pm 0.000044$ & $2993 \pm 13$ & $243 \pm 16$ & $5.9 \pm 0.6$ & $1.53 \pm 0.17$ & $10.657 \pm 0.045$ & $8.827 \pm 0.045$ \\
\hline
\end{tabular}

Notes. (1) Either the entire galaxy (aperture radius of 80") or the H I peak (see Fig. 3, aperture radius of 30"). (2) Redshift determined from the Gaussian fit to the H I spectrum. (3) The corresponding velocity. (4) Full width at half maximum of this Gaussian. (5) Peak of this Gaussian. (6) Integrated flux within $2 \sigma$ of the Gaussian width. (7) H I line luminosity using Eq. (3) in Solomon et al. (1997). (8) Neutral hydrogen mass using Eq. (2) in Devereux \& Young (1990).
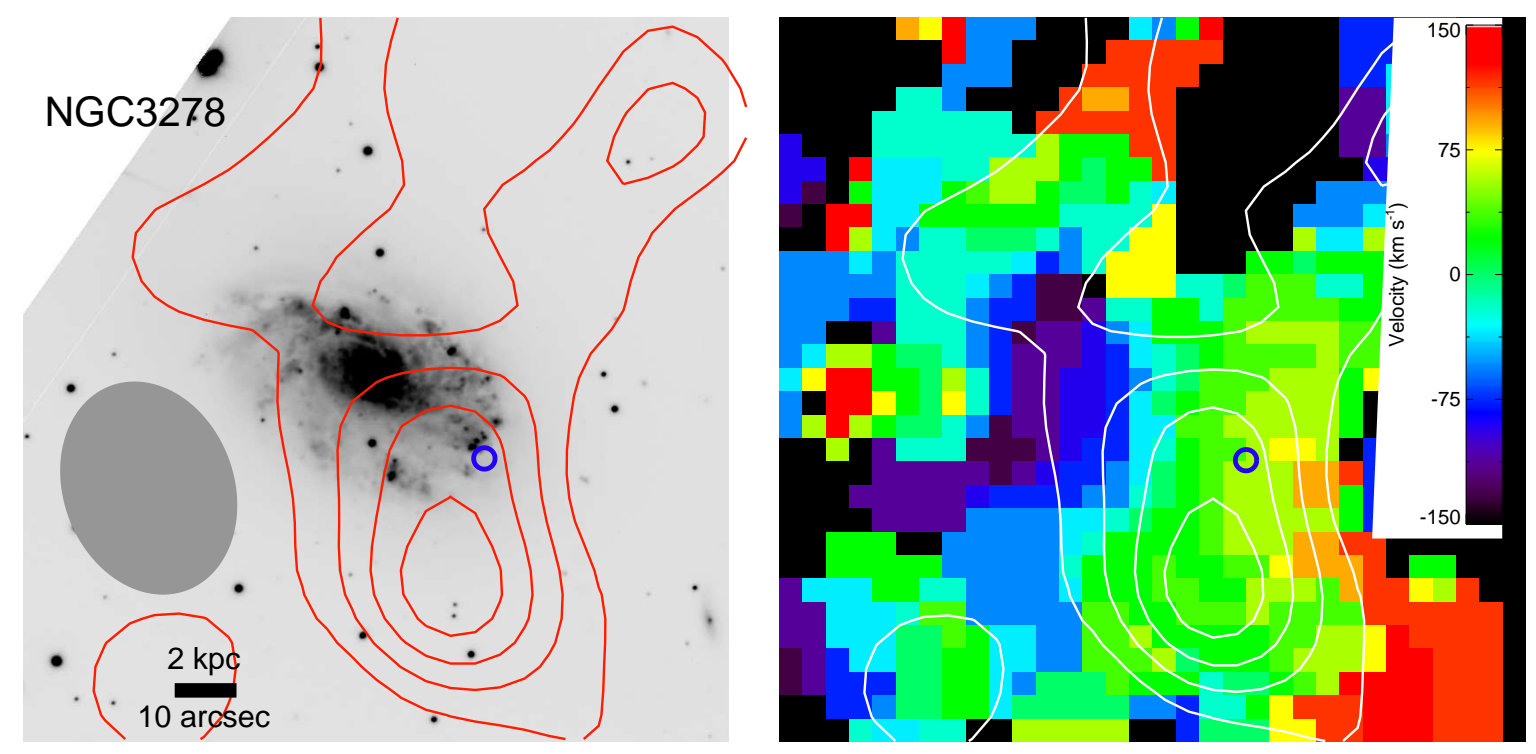

Fig. 3. Left: H I contours (red) of NGC 3278 on the optical $r$-band image of the galaxy (Pignata et al. 2011). The contours are $2,3,4,5 \sigma$, where $\sigma=0.17 \mathrm{Jy} \mathrm{beam}^{-1} \mathrm{~km} \mathrm{~s}^{-1}$ (corresponding to a neutral hydrogen column density of $\sim 1.9 \times 10^{20} \mathrm{~cm}^{2}$ ) is the rms of the collapsed image. The position of SN 2009bb is indicated by the blue circle. H I is concentrated close to this position. The beam size of the H I data is shown as the grey ellipse. The image is $120^{\prime \prime} \times 120^{\prime \prime}$ and the scale is indicated by the ruler. North is up and east is left. Right: the first moment map (velocity field) of the H I line. The image has the same size as the left one and the same contours and the SN position are shown. The velocities are relative to the systemic velocity of $2961 \mathrm{~km} \mathrm{~s}^{-1}$ derived from optical spectra.

They reported the linewidth at a flux level that is $50 \%$ of the mean flux averaged in channels within the wavelength range enclosing $90 \%$ of the total integrated flux, $W_{m 50}=292 \pm 11$ and $295 \mathrm{~km} \mathrm{~s}^{-1}$, respectively. This is slightly higher than the Full width at half maximum (FWHM) given in Table 4 because the Gaussian function does not represent the line profile accurately. Indeed the Gaussian FHWM reported by Roth et al. (1994) of $225 \mathrm{~km} \mathrm{~s}^{-1}$ is consistent with our result. Our estimate of the integrated flux (which does not involve assumptions on the line shape), agrees with $5.9 \pm 0.5 \mathrm{Jy} \mathrm{km} \mathrm{s}^{-1}$ and $6.9 \pm$ $3.3 \mathrm{Jy} \mathrm{km} \mathrm{s}^{-1}$ reported by Courtois et al. (2011) and Roth et al. (1994), respectively.

According to the SFR- $M_{\mathrm{HI}}$ scaling relation (Eq. (1) in Michałowski et al. 2015), NGC 3278 with SFR $\sim 3 M_{\odot} \mathrm{yr}^{-1}$ (Tables 2 and 3) should have $\log \left(M_{\mathrm{HI}} / M_{\odot}\right) \sim 10,0.7$ dex higher than the measured value. The scatter of this relation is significant $(0.38$ dex $1 \sigma)$, so this is not unusual, but we conclude that NGC 3278 exhibits low atomic gas content for its SFR.

The CO fluxes, luminosities, and the resulting molecular gas masses are presented in Table 5. The molecular gas mass based on the $\mathrm{CO}(2-1)$ line is a $\sim 0.15$ dex lower than that based on the $\mathrm{CO}(1-0)$, but this is very likely due to the beam size at the $\mathrm{CO}(2-1)$ transition of $24^{\prime \prime}$ being too small to cover the entire galaxy (Fig. A.1), so the corresponding $\mathrm{CO}(2-1)$ flux is underestimated. Therefore, only the estimates based on the $\mathrm{CO}(1-0)$ line are used in the following analysis.

Using the total infrared luminosity of $L_{\mathrm{IR}} \sim 5 \times 10^{10} L_{\odot}$ (Tables 2 and 3), we estimate the star formation efficiency (SFE) of $L_{\mathrm{IR}} / L_{\mathrm{CO}(1-0)}^{\prime} \sim 100 L_{\odot} /\left(\mathrm{K} \mathrm{km} \mathrm{s}^{-1} \mathrm{pc}^{2}\right)$. This is one of the highest numbers among local spirals with $\sim(48 \pm 7) L_{\odot} /\left(\mathrm{K} \mathrm{km} \mathrm{s}^{-1} \mathrm{pc}^{2}\right)$ derived by Daddi et al. (2010, their Fig. 13). Similarly, the relation between SFR, CO luminosity, and metallicity presented in Hunt et al. (2015, their Fig. 5), $\log \left(\mathrm{SFR} / L^{\prime} \mathrm{CO}\right)=-2.25 \times$ $[12+\log (\mathrm{O} / \mathrm{H})]+11.31$ predicts an $\mathrm{SFR} / L^{\prime}{ }_{\mathrm{CO}}$ of the SN 2009bb host of $\sim 3.5 \times 10^{-9} M_{\odot} \mathrm{yr}^{-1} /\left(\mathrm{K} \mathrm{km} \mathrm{s}^{-1} \mathrm{pc}^{2}\right)$, whereas the measured value is $\sim 1.5-2$ times higher, 5-6.5 $\times 10^{-9} M_{\odot} \mathrm{yr}^{-1} /\left(\mathrm{K} \mathrm{km} \mathrm{s}^{-1} \mathrm{pc}^{2}\right)$, indicating low $\mathrm{CO}$ luminosity for its SFR and metallicity. Hence, the SN 2009bb host galaxy has also a few times lower molecular gas content than its SFR would suggest. Molecular gas deficiency was also claimed for some GRB hosts (Hatsukade et al. 2014; Stanway et al. 2015b; Michałowski et al. 2016, 2018a, but we note that this result does not hold for the host galaxy of GRB 020819B, for which the initial host identification was proven to be wrong; see Perley et al. 2017). On the other hand, normal molecular gas properties were found in other GRB hosts 
Table 5. CO fluxes, luminosities, and molecular gas masses of NGC 3278, based on the data from Albrecht et al. (2007).

\begin{tabular}{lccccc}
\hline \hline $\begin{array}{l}\mathrm{CO} \\
\text { trans. }\end{array}$ & $\begin{array}{c}\text { FWHM } \\
\left({ }^{\prime \prime}\right)\end{array}$ & $\begin{array}{c}F_{\text {int }} \\
\left(\mathrm{Jy} \mathrm{km} \mathrm{s}^{-1}\right)\end{array}$ & $\begin{array}{c}\log \left(L_{\mathrm{CO}}^{\prime}\right) \\
\left(\mathrm{K} \mathrm{km} \mathrm{s}^{-1} \mathrm{pc}^{2}\right)\end{array}$ & $\begin{array}{c}\log \left(L_{\mathrm{CO}}\right) \\
\left(L_{\odot}\right)\end{array}$ & $\begin{array}{c}\log \left(M_{\mathrm{H}_{2}}\right) \\
\left(M_{\odot}\right)\end{array}$ \\
\hline $1-0$ & $(2)$ & $(3)$ & $(4)$ & $(5)$ & $(6)$ \\
$2-1$ & 45 & 132 & 8.75 & 4.41 & 9.45 \\
\hline
\end{tabular}

Notes. (1) CO transition. (2) Full width at half maximum of the telescope (green circles on Fig. A.1). (3) Integrated flux. (4) Line luminosity using Eq. (3) in Solomon et al. (1997). (5) Line luminosity in solar luminosity units. (6) Molecular hydrogen mass assuming CO-to- $\mathrm{H}_{2}$ conversion factor $\alpha_{\mathrm{CO}}=5 M_{\odot} /\left(\mathrm{K} \mathrm{km} \mathrm{s}^{-1} \mathrm{pc}^{2}\right)$. In order to calculate $M_{\mathrm{H}_{2}}$ from the 2-1 transition, we assumed $L_{\mathrm{CO}(1-0)}^{\prime}=2 \times L_{\mathrm{CO}(2-1)}^{\prime}$.

(Arabsalmani et al. 2018), with the current status that the sample on average does not deviate from other star-forming galaxies (Michałowski et al. 2018a).

We also used the relation between the metallicity, atomic gas, and molecular gas for dwarf galaxies provided by Filho et al. (2016, their Sect. 4), based on the calibration of Amorín et al. (2016): $\log \left(M_{\mathrm{H}_{2}}\right)=1.2 \log \left(M_{\mathrm{HI}}\right)-1.5 \times[12+\log (\mathrm{O} / \mathrm{H})-8.7]-$ 2.2. For its atomic gas mass (Table 4$)$ and average metallicity (Table A.1, last row using the calibration of Dopita et al. 2016), the SN 2009bb host should have $\log \left(M_{\mathrm{H}_{2}} / M_{\odot}\right) \sim 8.85$, approximately four times lower than the actual $\mathrm{CO}$ estimate (Table 5). NGC 3278 would be consistent with this relation if it had a much lower metallicity of $12+\log (\mathrm{O} / \mathrm{H}) \sim 8.4$ (half solar where solar metallicity is $12+\log (\mathrm{O} / \mathrm{H}) \sim 8.66$; Asplund et al. 2004).

We find that the SN 2009bb host has a molecular gas mass fraction of $M_{\mathrm{H}_{2}} /\left(M_{\mathrm{H}_{2}}+M_{\mathrm{HI}}\right) \sim 57 \%$, which is high, but within the range for other star-forming galaxies (a percentage of a few to a few tens; Young et al. 1989; Devereux \& Young 1990; Leroy et al. 2008; Saintonge et al. 2011; Cortese et al. 2014; Boselli et al. 2014), and of hosts of $\mathrm{SNe}$ of different type (Galbany et al. 2017).

\subsection{Resolved ISM and stellar properties}

Figure 4 shows the $1.4 \mathrm{GHz}$ continuum image from Condon et al. (1996). The emission is lopsided and the peak of the emission is close to the position of SN 2009bb.

Based on the MUSE observations, the distribution of $\mathrm{H} \alpha$ flux, equivalent width (EW), SFR, and the velocity field is shown in Fig. 5 and the distribution of dust extinction and metallicity is shown in Fig. 6. We derived SFRs of each spaxel from the $\mathrm{H} \alpha$ fluxes using the calibration of Kennicutt (1998) with the Chabrier (2003) IMF. The dust extinction was derived from the Balmer decrement. We made three metallicity measurements based on [SII], [NII], and $\mathrm{H} \alpha$ fluxes (Dopita et al. 2016, used in all analysis unless stated otherwise), [OIII], [NII], $\mathrm{H} \alpha$, and $\mathrm{H} \beta$ lines (O3N2), and just [NII] and $\mathrm{H} \alpha(\mathrm{N} 2)$ lines (Pettini \& Pagel 2004).

The properties of $\mathrm{H} \alpha$-detected star-forming regions were extracted in apertures with radius of $0.5^{\prime \prime}(\sim 100 \mathrm{pc})$ from these maps, shown in Fig. 7 as a function of a deprojected galactocentric distance (and in Fig. A.2 using measured instead of deprojected distances), and listed in Table A.1. They were visually selected in the $\mathrm{H} \alpha$ map down to approximately $10^{-16} \mathrm{erg} \mathrm{s}^{-1} \mathrm{~cm}^{-2}$. This corresponds to the $\mathrm{H} \alpha$ luminosity of $\sim 2 \times 10^{37} \mathrm{erg} \mathrm{s}^{-1}$ at the redshift of NGC 3278, which is comparable to the luminosity of H II regions in the Milky Way and nearby galaxies (e.g. Crowther 2013). The first row in Table A.1 is the SN 2009bb position and the second is the centre of the galaxy. The last row shows the

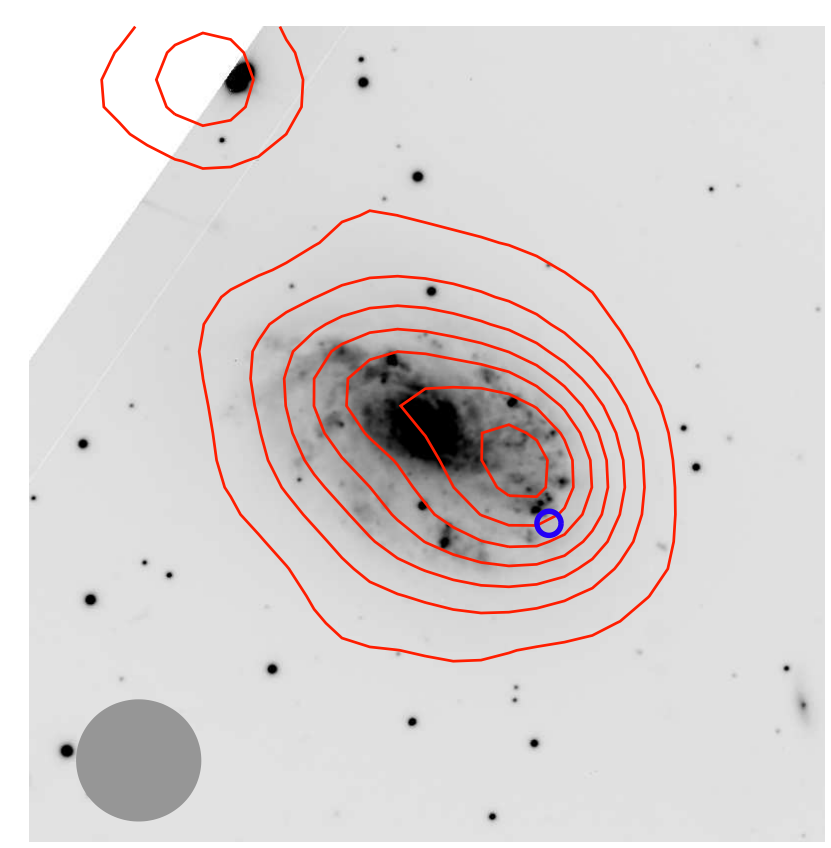

Fig. 4. Continuum 1.4 GHz contours (red; from Condon et al. 1996) of NGC 3278 on the optical $r$-band image of the galaxy (Pignata et al. 2011). The lowest contour is at $1 \mathrm{mJybeam}^{-1}$ and the step is $2 \mathrm{mJy}$ beam $^{-1}$. The position of SN 2009bb is indicated by the blue circle. Radio continuum emission peaks close to this position. The beam size of the radio data is shown as the grey circle. The image is $120^{\prime \prime} \times 120^{\prime \prime}$. North is up and east is left.

sum of the individual regions for extensive properties ( $\mathrm{H} \alpha$ flux and SFR) and the average for the intensive properties (equivalent width, extinction, and metallicities). The parameters of the linear fit of the properties as a function of distance from the galaxy centre are presented in Table 6 . The $\mathrm{SN}$ region is one of the most star-forming regions within its host in terms of both SFR and sSFR.

The metallicities at the $\mathrm{SN}$ position and the nearest bright star-forming region (the fifth row) are $\sim 0.2-0.4 \mathrm{dex}$ lower than the value of $12+\log (\mathrm{O} / \mathrm{H}) \sim 8.96 \pm 0.10$ reported by Levesque et al. (2010c). This is because they used the [NII]/[OIII] method of Kewley \& Dopita (2002), which was shown to result in systematically higher metallicities than the methods we employed (Kewley \& Ellison 2008). Indeed using the fluxes reported by Levesque et al. (2010c) for the $\mathrm{SN}$ region, we obtained $12+\log (\mathrm{O} / \mathrm{H}) \sim 8.68$ and $\sim 8.63$, for the O3N2 and N2 methods of Pettini \& Pagel (2004), consistent with our results. The velocity field derived from the $\mathrm{H} \alpha$ line (Fig. 5) is typical for a rotating disk galaxy. 

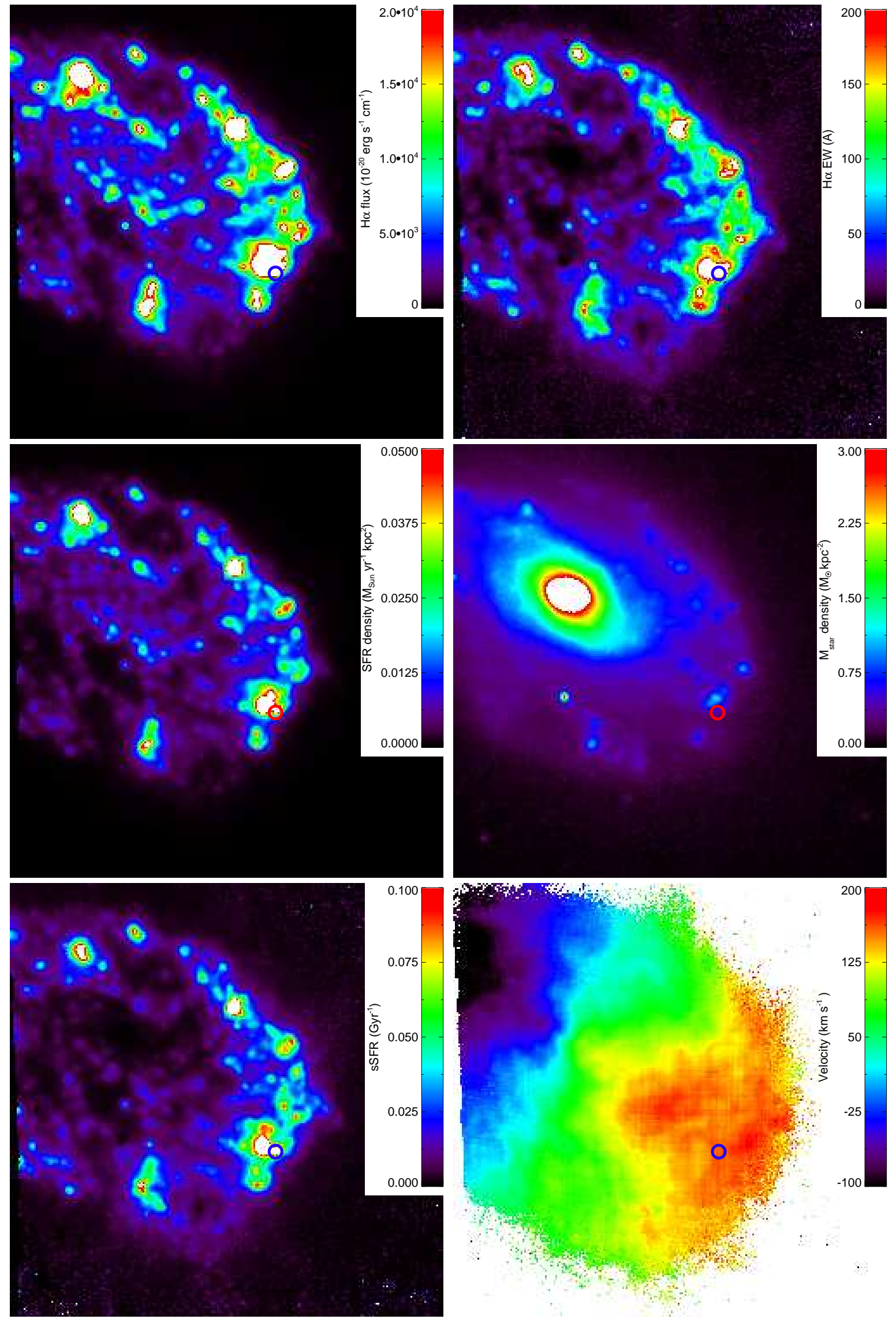

Fig. 5. MUSE maps: $\mathrm{H} \alpha$ flux, equivalent width, SFR density from $\mathrm{H} \alpha$ flux, stellar mass density from $H$-band, specific SFR, and velocity field. The position of SN 2009bb is indicated by the blue or red circle. The images are 50" $\times 50^{\prime \prime}$ (not the entire MUSE coverage). North is up and east is left. White indicates values above the maximum value in the colour bars. The velocities are relative to the systemic velocity of $2961 \mathrm{~km} \mathrm{~s}^{-1}$. 

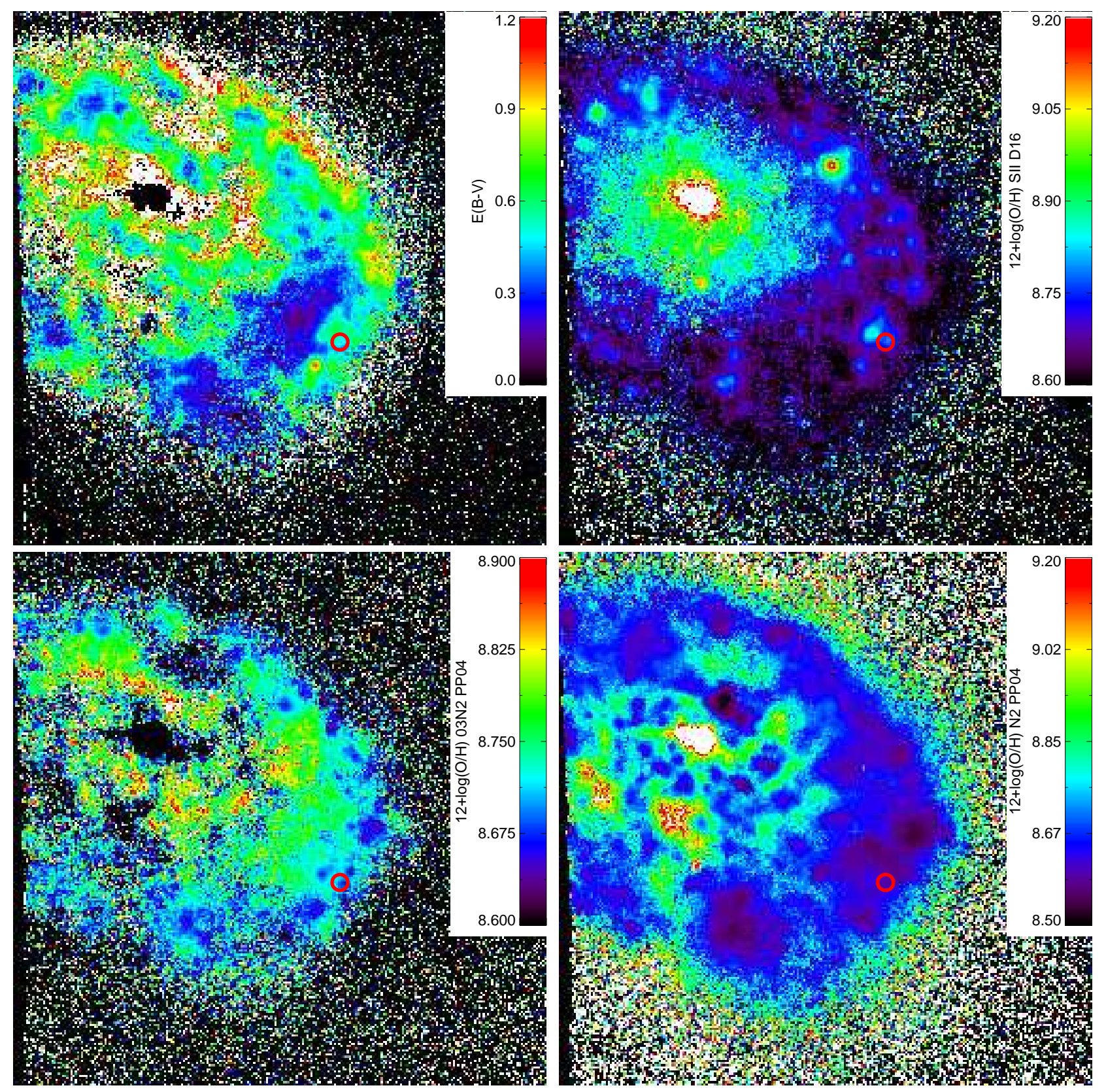

Fig. 6. Same as for Fig. 5, but for extinction and the metallicity indicators based on [SII], [NII], and H $\alpha$ fluxes (Dopita et al. 2016), [OIII], [NII], $\mathrm{H} \alpha$, and $\mathrm{H} \beta$ lines (Pettini \& Pagel 2004), and on [NII] and $\mathrm{H} \alpha$ lines (Pettini \& Pagel 2004).

\section{Discussion}

In summary, NGC 3278 has an enhanced SFR given its stellar mass (close to the starburst regime above the main sequence, Sect. 4.1), low atomic and molecular gas masses given its SFR (Sect. 4.2), and the $\mathrm{SN}$ region is one of the most star-forming regions (Figs. 4 and 7). The atomic gas distribution is not centred on the optical galaxy centre, but instead around a third of atomic gas resides in the region close to the SN position (Fig. 3). These properties are similar to the H I concentrations close to the GRB positions (Michałowski et al. 2015; Arabsalmani et al. 2015) and to the claimed molecular deficiency of GRB hosts (Hatsukade et al. 2014; Stanway et al. 2015b; Michałowski et al. 2016). This was interpreted in Michałowski et al. (2015, 2016) as an indication that a very recent inflow of metal-poor atomic gas is responsible for enhanced SFRs, and, in turn, for the birth of the GRB progenitors. This is likely the case for SN 2009bb. The fact that we executed the high-resolution ATCA H I observations of a relativistic SN host and obtained a similar unusual distribution suggests that both explosion classes prefer similar environments. This needs to be tested with a larger sample of SN hosts observed at HI. The recent inflow of gas for NGC 3278 is also supported by the relatively low metallicity measured in the southwestern part of the galaxy (Fig. 6), close to the Hi peak.

The H I velocity field also points at the external origin of at least some of the atomic gas. The $\mathrm{H}$ I velocity field (Fig. 3 ) is not consistent with a rotating disk, as opposed to the $\mathrm{H} \alpha$ velocity field (Fig. 5). Moreover, the values of velocities derived from the $\mathrm{H}$ I and $\mathrm{H} \alpha$ lines are not consistent at the same positions. For example, close to the SN position the $\mathrm{H} \alpha$ line gives $\sim 200 \mathrm{~km} \mathrm{~s}^{-1}$, whereas the $\mathrm{H}$ I line results in $\sim 50 \mathrm{~km} \mathrm{~s}^{-1}$. 


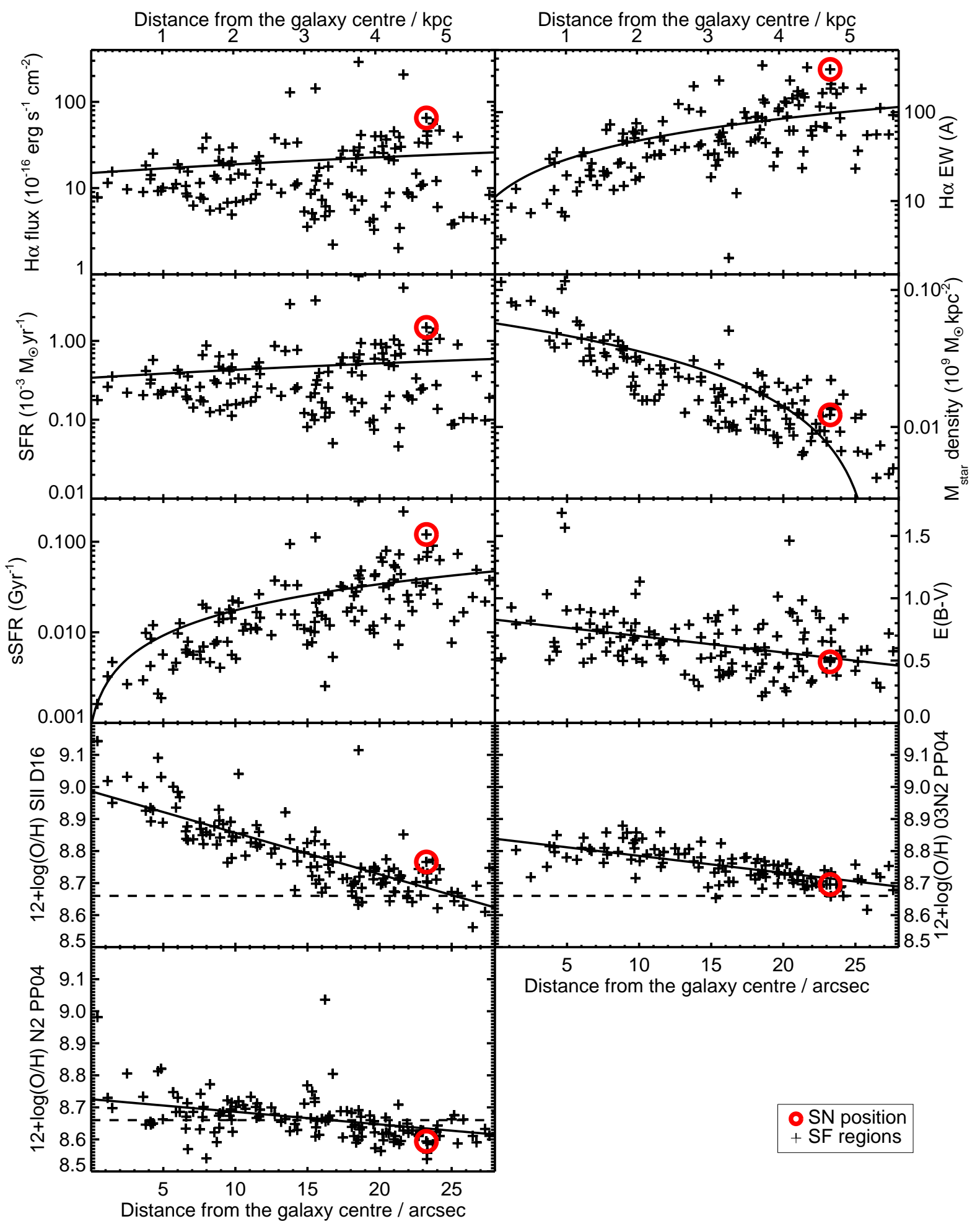

Fig. 7. Properties of $\mathrm{H} \alpha$-selected star-forming regions as a function of deprojected distance from the galaxy centre (Sect. 2.3): H $\alpha$ flux, equivalent width, SFR from $\mathrm{H} \alpha$ flux, stellar mass density, specific SFR, extinction, and three metallicity measurements based on [SII], [NII], and H $\alpha$ fluxes (Dopita et al. 2016), [OIII], [NII], $\mathrm{H} \alpha$, and $\mathrm{H} \beta$ lines, and just [NII] and $\mathrm{H} \alpha$ lines (Pettini \& Pagel 2004). The region in which SN $2009 \mathrm{bb}$ exploded is indicated by red circles. The linear fits to the data (Table 6$)$ are shown as solid lines. The solar metallicity of $12+\log (\mathrm{O} / \mathrm{H}) \sim 8.66($ Asplund et al. 2004) is marked as a dashed line.

SN 2009bb exploded close to the region with the highest SFR density and the lowest age, as evident from the high $\mathrm{H} \alpha$ EW (Figs. 5 and 7), similarly to other SN Ib/c (Galbany et al. 2014) and SN II (Galbany et al. 2016a). Following Kuncarayakti (2013a,b) we converted the $\mathrm{H} \alpha \mathrm{EW}$ of the SN site of $\sim 300 \AA$ to the stellar population age of $\sim 5.5 \mathrm{Myr}$ (assuming instantaneous burst and standard Salpeter 1955 IMF) by comparing to single stellar population models from Starburst99 (Leitherer et al. 1999). This timescale corresponds to the lifetime of a $\sim 36 M_{\odot}$ massive star. Although it is not straightforward to infer this as 
Table 6. Linear fit of the properties as a function of distance from the galaxy centre (Fig. 7) in the form $A+B_{1} \times$ dist $_{\text {arcsec }}$ or $A+B_{2} \times$ dist $_{\mathrm{kpc}}$. The variables and the units are as in Table A.1.

\begin{tabular}{lccc}
\hline \hline Property & $\begin{array}{c}A \\
\text { (unit) }\end{array}$ & $\begin{array}{c}B_{1} \\
\text { (unit/arcsec) }\end{array}$ & $\begin{array}{c}B_{2} \\
\text { (unit/kpc) }\end{array}$ \\
\hline HaFlux & 11.8 & 0.72 & 3.56 \\
HaEW & 7.6 & 4.8 & 23.6 \\
SFR & 0.27 & 0.0165 & 0.081 \\
Mass & 0.05 & -0.0023 & -0.011 \\
SSFR & -0.00112 & 0.0022 & 0.011 \\
ebv & 0.84 & -0.017 & -0.086 \\
OH_D16 & 8.97 & -0.015 & -0.072 \\
OH_PPQ4_03N2 & 8.83 & -0.006 & -0.028 \\
OH_PPQ4_N2 & 8.73 & -0.005 & -0.025 \\
\hline
\end{tabular}

the initial mass of the SN 2009bb progenitor, the fact that such a young age is observed at the explosion site supports the view that the progenitor may have been one of such massive stars. This also means that in the scenario of the gas inflow presented above, it must have begun only several Myr ago, consistent with the timescale presented in Michałowski et al. (2016) for a GRB host.

The metallicity of the site of SN $2009 b b(12+\log (\mathrm{O} / \mathrm{H}) \sim$ 8.77 or $\sim 1.3$ solar using the calibration of Dopita et al. 2016; Table A.1) is close to the highest values found for other SN Ib/c (Thöne et al. 2009; Leloudas et al. 2011; Kuncarayakti 2013a; Kuncarayakti et al. 2018; Galbany et al. 2016b) and II-L (Kuncarayakti 2013b). On the other hand, GRBs are usually found in environments with much lower metallicities (Sollerman et al. 2005; Christensen et al. 2008; Modjaz et al. 2008; Thöne et al. 2008, 2014; Han et al. 2010; Levesque et al. 2010a, 2011; Krühler et al. 2015, 2017; Schulze et al. 2015; Japelj et al. 2016; Izzo et al. 2017; Vergani et al. 2017). However, there is a growing sample of GRBs in solar or super-solar environments (Prochaska et al. 2009; Levesque et al. 2010b; Krühler et al. 2012; Savaglio et al. 2012; Elliott et al. 2013; Schulze et al. 2014; Hashimoto et al. 2015; Schady et al. 2015; Stanway et al. 2015a; Michałowski et al. 2018b), which can be explained by recent overcoming of the observational bias against dust, resulting in the discovery of massive and metal-rich hosts (Hjorth et al. 2012; Perley et al. 2015, 2016a,b). This metallicity information may mean that relativistic explosions signal a recent inflow of gas (and subsequent star formation), and their type (GRBs or $\mathrm{SNe}$ ) is determined by either (i) the metallicity of the inflowing gas, so that metal-poor gas results in a GRB explosion and metal-rich gas ${ }^{7}$ in a relativistic SN explosion without an accompanying GRB (see also Modjaz et al. 2011; Leloudas et al. 2010, 2011), or (ii) by the efficiency of gas mixing (efficient mixing for $\mathrm{SN}$ hosts leading to quick disappearance of metal-poor regions), or (iii) by the type of the galaxy (more metal-rich galaxies would result in only a small fraction of star formation being fuelled by metal-poor gas).

Stott et al. (2014) interpreted flat metallicity gradients as a sign of a recent inflow of metal-poor gas, because these gradients correlate with sSFR and the distance above the main sequence

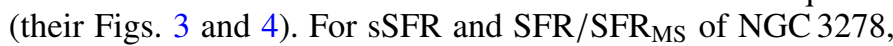

\footnotetext{
This would either be a minor merger with an evolved galaxy or gas coming from nearby galaxies, or previously ejected gas falling back Below is stated the evidence of the existence of a galaxy group close to NGC 3278.
}

their relation predicts the metallicity gradient of $\sim-0.02$ and $-0.01 \mathrm{dex} \mathrm{kpc}^{-1}$, respectively, using the N2 calibrator. This is similar to the $-0.019 \mathrm{dex} \mathrm{kpc}^{-1}$ measured for NGC 3278 using this calibrator (Table 6). Moreover, the gradients of -0.06 and $-0.03 \mathrm{dex} \mathrm{kpc}^{-1}$ for the hosts of GRB 980425 (Krühler et al. 2017) and 060505 (Thöne et al. 2008), respectively, are also consistent with the trends of Stott et al. (2014), using the sSFR values tabulated in Michałowski et al. (2015). This therefore needs to be investigated with a larger sample of SN and GRB hosts. If these galaxies turn out to have steeper metallicity gradients, this would support the scenario that the gas inflow is of higher metallicity or smaller in quantity, so it does not flatten the metallicity gradient.

Finally, the distributions of the $\mathrm{H} \alpha$ emission (Fig. 5), of the optical emission, and especially the radio continuum emission (Fig. 4) of NGC 3278 are clearly lopsided, with the western (right) side more pronounced and rich in star-forming regions. Such asymmetry may be a sign of interaction (Sancisi et al. 2008; Rasmussen et al. 2006), so we looked at the large-scale environment of NGC 3278 using the NASA/IPAC Extragalactic Database (NED). We found a galaxy group designated 0509 in Tully et al. (2008) at coordinates 10:27:10.4, $-39: 52: 58, \sim 51^{\prime}$ or $\sim 600 \mathrm{kpc}$ west in projection from NGC 3278 with $z=$ 0.009493 (similar to the redshift of NGC 3278 , shifted only by $\sim 100 \mathrm{~km} \mathrm{~s}^{-1}$ ). The distance of $600 \mathrm{kpc}$ may be too high to influence NGC 3278 that strongly (e.g. the clear sign of interaction reported by Rasmussen et al. 2006 concerns a galaxy $\sim 70 \mathrm{kpc}$ from the group). However the existence of the galaxy group in the vicinity of NGC 3278 indicates that indeed there should be a significant supply of ambient intergalactic gas available for inflow onto this galaxy.

The caveat of this work is that SN 2009bb was discovered in a galaxy-targeted survey. Such surveys were shown to result on average in higher metallicities and masses than un-targeted surveys (Sanders et al. 2012). Therefore analysis of the gas content of a larger sample of relativistic SN hosts from both targeted and un-targeted surveys is needed. We note, however, that hosts of broad-line Ic SN from both targeted and un-targeted surveys do include objects with metallicities around solar (Sanders et al. 2012), similar to NGC 3278 .

\section{Conclusions}

We obtained $21 \mathrm{~cm}$ hydrogen line (H I) and optical integral field unit spectroscopy observations of NGC 3278, the host galaxy of the relativistic SN2009bb. This is the first time the atomic gas properties of a relativistic SN host have been provided and the first time resolved $21 \mathrm{~cm}$-hydrogen-line $(\mathrm{HI})$ information is analysed for the host of an SN of any type in the context of the SN position. The atomic gas distribution of NGC 3278 is not centred on the optical galaxy centre, but instead around a third of the atomic gas resides in the region close to the $\mathrm{SN}$ position. This galaxy has a few times lower atomic and molecular gas masses than predicted from its SFR. Its specific SFR $\left(\mathrm{sSFR} \equiv \mathrm{SFR} / M_{*}\right)$ is approximately two to three times higher than the main-sequence value, placing it at the higher end of the main sequence towards starburst galaxies. SN 2009bb exploded close to the region with the highest SFR density and the lowest age, as evident from a high $\mathrm{H} \alpha \mathrm{EW}$, corresponding to the age of the stellar population of $~ 5.5 \mathrm{Myr}$. Assuming this timescale was the lifetime of the progenitor star, its initial mass would have been close to $\sim 36 M_{\odot}$. The gas properties of NGC 3278 are consistent with a recent inflow of gas from the intergalactic medium, which explains the concentration of atomic gas close to the SN 
position and the enhanced SFR. Super-solar metallicity at the position of the SN (unlike for most GRBs) may mean that relativistic explosions signal a recent inflow of gas (and subsequent star formation), and their type (GRBs or SNe) is determined by either (i) the metallicity of the inflowing gas, so that metal-poor gas results in a GRB explosion and metal-rich gas (for example a minor merger with an evolved galaxy or re-accretion of expelled gas) in a relativistic SN explosion without an accompanying GRB, (ii) the efficiency of gas mixing (efficient mixing for SN hosts leading to quick disappearance of metal-poor regions), or (iii) the type of the galaxy (more metal-rich galaxies would result in only a small fraction of star formation being fuelled by metal-poor gas).

Acknowledgements. We thank Joanna Baradziej for help to improve this paper and Giuliano Pignata, Carlos Contreras, and Maximilian Stritzinger for sharing the $H$-band image. M.J.M. acknowledges the support of the National Science Centre, Poland, through the POLONEZ grant 2015/19/P/ST9/04010 and the UK Science and Technology Facilities Council; this project has received funding from the European Union's Horizon 2020 research and innovation programme under the Marie Skłodowska-Curie grant agreement No. 665778 J.H. was supported by a VILLUM FONDEN Investigator grant (project number 16599). L.G. was supported in part by the US National Science Foundation under Grant AST-1311862. L.K.H. acknowledges funding from the INAF PRIN-SKA program 1.05.01.88.04. A.d.U.P. acknowledges support from the European Commission (FP7-PEOPLE-2012-CIG 322307) and from the Spanish project AYA2012-39362-C02-02. S.D.V. is supported by the French National Research Agency (ANR) under contract ANR-16-CE31-0003 BEaPro. The Australia Telescope Compact Array is part of the Australia Telescope National Facility, which is funded by the Commonwealth of Australia for operation as a National Facility managed by CSIRO. Based on observations collected at the European Organisation for Astronomical Research in the Southern Hemisphere under ESO programme(s) 095.D-0172(A). This publication makes use of data products from the Wide-field Infrared Survey Explorer, which is a join project of the University of California, Los Angeles, and the Jet Propulsion Laboratory/California Institute of Technology, funded by the National Aeronautics and Space Administration. We acknowledge the usage of the HyperLeda database (http://leda.univ-lyon1.fr). This research has made use of the GHostS database (http://www.grbhosts.org), which is partly funded by Spitzer/NASA grant RSA Agreement No. 1287913; the NASA/IPAC Extragalactic Database (NED), which is operated by the Jet Propulsion Laboratory, California Institute of Technology, under contract with the National Aeronautics and Space Administration; SAOImage DS9, developed by Smithsonian Astrophysical Observatory (Joye \& Mandel 2003); and NASA's Astrophysics Data System Bibliographic Services.

\section{References}

Albrecht, M., Krügel, E., \& Chini, R. 2007, A\&A, 462, 575

Amorín, R., Muñoz-Tuñón, C., Aguerri, J. A. L., \& Planesas, P. 2016, A\&A, 588, A 23

Arabsalmani, M., Roychowdhury, S., Zwaan, M. A., Kanekar, N., \& Michałowski, M. J. 2015, MNRAS, 454, L51

Arabsalmani, M., Le Floc'h, E., Dannerbauer, H., et al. 2018, MNRAS, 476, 2332

Asplund, M., Grevesse, N., Sauval, A. J., Allende Prieto, C., \& Kiselman, D. 2004, A\&A, 417, 751

Bacon, R., Accardo, M., Adjali, L., et al. 2010, Proc. SPIE, 7735, 773508

Bietenholz, M. F., Soderberg, A. M., Bartel, N., et al. 2010, ApJ, 725, 4

Bigiel, F., Leroy, A., Walter, F., et al. 2008, AJ, 136, 2846

Bigiel, F., Leroy, A., Walter, F., et al. 2010, AJ, 140, 1194

Boselli, A., Cortese, L., \& Boquien, M. 2014, A\&A, 564, A65

Bruzual, G., \& Charlot, S. 2003, MNRAS, 344, 1000

Carilli, C. L., \& Walter, F. 2013, MNRAS, 51, 105

Castro Cerón, J. M., Michałowski, M. J., Hjorth, J., et al. 2010, ApJ, 721, 1919 Chabrier, G. 2003, ApJ, 586, L133

Chakraborti, S., Soderberg, A., Chomiuk, L., et al. 2015, ApJ, 805, 187

Christensen, L., Vreeswijk, P. M., Sollerman, J., et al. 2008, A\&A, 490, 45

Condon, J. J., Helou, G., Sanders, D. B., \& Soifer, B. T. 1996, ApJS, 103, 81

Condon, J. J., Cotton, W. D., Greisen, E. W., et al. 1998, AJ, 115, 1693

Cortese, L., Fritz, J., Bianchi, S., et al. 2014, MNRAS, 440, 942

Courtois, H. M., Tully, R. B., Makarov, D. I., et al. 2011, MNRAS, 414, 2005

Crowther, P. A. 2013, MNRAS, 428, 1927

da Cunha, E., Charlot, S., \& Elbaz, D. 2008, MNRAS, 388, 1595
Daddi, E., Bournaud, F., Walter, F., et al. 2010, ApJ, 713, 686

De Colle, F., Kumar, P., Aguilera-Dena, D. R., 2018, ApJ, 863, 32

de Vaucouleurs, G., de Vaucouleurs, A., Corwin, H. G., Jr., et al. 1991, Third

Reference Catalogue of Bright Galaxies (New York: Springer)

Devereux, N. A., \& Young, J. S. 1990, ApJ, 359, 42

Dopita, M. A., Kewley, L. J., Sutherland, R. S., \& Nicholls, D. C. 2016, Ap\&SS, 361,61

Draine, B. T., \& Lee, H. M. 1984, ApJ, 285, 89

Elliott, J., Krühler, T., Greiner, J., et al. 2013, A\&A, 556, A23

Elmegreen, B. G., Kaufman, M., Bournaud, F., et al. 2016, ApJ, 823, 26

Filho, M. E., Sánchez Almeida, J., Amorín, R., et al. 2016, ApJ, 820, 109

Fumagalli, M., \& Gavazzi, G. 2008, A\&A, 490, 571

Galbany, L., Stanishev, V., Mourão, A. M., et al. 2014, A\&A, 572, A38

Galbany, L., Anderson, J. P., Rosales-Ortega, F. F., et al. 2016a, MNRAS, 455, 4087

Galbany, L., Stanishev, V., Mourão, A. M., et al. 2016b, A\&A, 591, A48

Galbany, L., Mora, L., González-Gaitán, S., et al. 2017, MNRAS, 468, 628

Glover, S. C. O., \& Clark, P. C. 2012, MNRAS, 421, 9

Han, X. H., Hammer, F., Liang, Y. C., et al. 2010, A\&A, 514, A24

Hashimoto, T., Perley, D. A., Ohta, K., et al. 2015, ApJ, 806, 250

Hatsukade, B., Ohta, K., Endo, A., et al. 2014, Nature, 510, 247

Hjorth, J., Malesani, D., Jakobsson, P., et al. 2012, ApJ, 756, 187

Hu, C. Y., Naab, T., Walch, S., Glover, S. C. O., \& Clark, P. C. 2016, MNRAS, 458, 3528

Hunt, L. K., García-Burillo, S., Casasola, V., et al. 2015, A\&A, 583, A114

Iglesias-Páramo, J., Buat, V., Hernández-Fernández, J., et al. 2007, ApJ, 670, 279

Izzo, L., Thöne, C. C., Schulze, S., et al. 2017, MNRAS, 472, 4480

Japelj, J., Vergani, S. D., Salvaterra, R., et al. 2016, A\&A, 590, A129

Jarrett, T. H., Chester, T., Cutri, R., et al. 2000, AJ, 119, 2498

Joye, W. A., \& Mandel, E. 2003, in Astronomical Data Analysis Software and Systems XII, eds. H. E. Payne, R. I. Jedrzejewski, \& R. N. Hook, ASP Conf. Ser., 295, 489

Kennicutt, R. C. 1998, MNRAS, 36, 189

Kewley, L. J., \& Dopita, M. A. 2002, ApJS, 142, 35

Kewley, L. J., \& Ellison, S. L. 2008, ApJ, 681, 1183

Krajnović, D., Cappellari, M., de Zeeuw, P. T., \& Copin, Y. 2006, MNRAS, 366, 787

Krühler, T., Fynbo, J. P. U., Geier, S., et al. 2012, A\&A, 546, A8

Krühler, T., Malesani, D., Fynbo, J. P. U., et al. 2015, A\&A, 581, A125

Krühler, T., Kuncarayakti, H., Schady, P., et al. 2017, A\&A, 602, A85

Krumholz, M. R. 2012, ApJ, 759, 9

Kuncarayakti, H., Doi, M., Aldering, G., et al. 2013a, AJ, 146, 30

Kuncarayakti, H., Doi, M., Aldering, G., et al. 2013b, AJ, 146, 31

Kuncarayakti, H., Anderson, J. P., Galbany, L., et al. 2018, A\&A, 613, A35

Lauberts, A., \& Valentijn, E. A. 1989, The Surface Photometry Catalogue of the ESO-Uppsala Galaxies (European Southern Observatory)

Lazzati, D., Morsony, B. J., Blackwell, C. H., \& Begelman, M. C. 2012, ApJ, 750,68

Leitherer, C., Schaerer, D., Goldader, J. D., et al. 1999, ApJS, 123, 3

Leloudas, G., Sollerman, J., Levan, A. J., et al. 2010, A\&A, 518, A29

Leloudas, G., Gallazzi, A., Sollerman, J., et al. 2011, A\&A, 530, A95

Leroy, A. K., Walter, F., Brinks, E., et al. 2008, AJ, 136, 2782

Levesque, E. M., Berger, E., Kewley, L. J., \& Bagley, M. M. 2010a, AJ, 139, 694

Levesque, E. M., Kewley, L. J., Graham, J. F., \& Fruchter, A. S. 2010b, ApJ, 712, L26

Levesque, E. M., Soderberg, A. M., Foley, R. J., et al. 2010c, ApJ, 709, L26

Levesque, E. M., Berger, E., Soderberg, A. M., \& Chornock, R. 2011, ApJ, 739, 23

Makarov, D., Prugniel, P., Terekhova, N., Courtois, H., \& Vauglin, I. 2014, A\&A, 570, A13

Margutti, R., Milisavljevic, D., Soderberg, A. M., et al. 2014, ApJ, 797, 107

Mauch, T., Murphy, T., Buttery, H. J., et al. 2003, MNRAS, 342, 1117

Michałowski, M. J., Hjorth, J., Castro Cerón, J. M., \& Watson, D. 2008, ApJ, 672,817

Michałowski, M. J., Hjorth, J., Malesani, D., et al. 2009, ApJ, 693, 347

Michałowski, M. J., Hjorth, J., \& Watson, D. 2010a, A\&A, 514, A67

Michałowski, M. J., Watson, D., \& Hjorth, J. 2010b, ApJ, 712, 942

Michałowski, M. J., Dunlop, J. S., Cirasuolo, M., et al. 2012, A\&A, 541, A85

Michałowski, M. J., Hayward, C. C., Dunlop, J. S., et al. 2014a, A\&A, 571, A75

Michałowski, M. J., Hunt, L. K., Palazzi, E., et al. 2014b, A\&A, 562, A70

Michałowski, M. J., Gentile, G., Hjorth, J., et al. 2015, A\&A, 582, A78

Michałowski, M. J., Castro Cerón, J. M., Wardlow, J. L., et al. 2016, A\&A, 595, A72

Michałowski, M. J., Karska, A., Rizzo, J. R., et al. 2018a, A\&A, 617, A143

Michałowski, M. J., Xu, D., Stevens, J., et al. 2018b, A\&A, 616, A169

Milisavljevic, D., Margutti, R., Parrent, J. T., et al. 2015, ApJ, 799, 51

Modjaz, M., Kewley, L., Kirshner, R. P., et al. 2008, AJ, 135, 1136 
Modjaz, M., Kewley, L., Bloom, J. S., et al. 2011, ApJ, 731, L4 Moffett, A. J., Lange, R., Driver, S. P., et al. 2016, MNRAS, 462, 4336 Paragi, Z., Taylor, G. B., Kouveliotou, C., et al. 2010, Nature, 463, 516 Perley, D. A., Perley, R. A., Hjorth, J., et al. 2015, ApJ, 801, 102 Perley, D. A., Krühler, T., Schulze, S., et al. 2016a, ApJ, 817, 7 Perley, D. A., Tanvir, N. R., Hjorth, J., et al. 2016b, ApJ, 817, 8 Perley, D. A., Krühler, T., Schady, P., et al. 2017, MNRAS, 465, L89 Pettini, M., \& Pagel, B. E. J. 2004, MNRAS, 348, L59

Pignata, G., Maza, J., Antezana, R., et al. 2009a, AIP Conf. Ser., 1111, 551

Pignata, G., Maza, J., Hamuy, M., et al. 2009b, Cent. Bureau Electron. Tel., 1731

Pignata, G., Stritzinger, M., Soderberg, A., et al. 2011, ApJ, 728, 14

Prochaska, J. X., Sheffer, Y., Perley, D. A., et al. 2009, ApJ, 691, L27

Rafelski, M., Gardner, J. P., Fumagalli, M., et al. 2016, ApJ, 825, 87

Rasmussen, J., Ponman, T. J., \& Mulchaey, J. S. 2006, MNRAS, 370, 453

Rossi, A., Piranomonte, S., Savaglio, S., et al. 2014, A\&A, 572, A47

Roth, J., Mould, J., \& Staveley-Smith, L. 1994, AJ, 108, 851

Saintonge, A., Kauffmann, G., Kramer, C., et al. 2011, MNRAS, 415, 32

Salpeter, E. E. 1955, ApJ, 121, 161

Sancisi, R., Fraternali, F., Oosterloo, T., \& van der Hulst, T. 2008, A\&ARv, 15, 189

Sanders, D. B., Mazzarella, J. M., Kim, D. C., Surace, J. A., \& Soifer, B. T. 2003, AJ, 126, 1607

Sanders, N. E., Soderberg, A. M., Levesque, E. M., et al. 2012, ApJ, 758, 132

Sault, R. J., \& Killeen, N. E. B. 2004, The Miriad User's Guide (Sydney: Australia Telescope National Facility), www.atnf.csiro.au/computing/ software/miriad/

Sault, R. J., Teuben, P. J., \& Wright, M. C. H. 1995, in Astronomical Data Analysis Software and Systems IV, eds. R. A. Shaw, H. E. Payne, \& J. J. E. Hayes, ASP Conf. Ser., 77, 433

Savaglio, S., Glazebrook, K., \& LeBorgne, D. 2009, ApJ, 691, 182

Savaglio, S., Rau, A., Greiner, J., et al. 2012, MNRAS, 420, 627

Schady, P., Krühler, T., Greiner, J., et al. 2015, A\&A, 579, A126

Schlafly, E. F., \& Finkbeiner, D. P. 2011, ApJ, 737, 103

Schulze, S., Malesani, D., Cucchiara, A., et al. 2014, A\&A, 566, A102

Schulze, S., Chapman, R., Hjorth, J., et al. 2015, ApJ, 808, 73

Silva, L., Granato, G. L., Bressan, A., \& Danese, L. 1998, ApJ, 509, 103

Skrutskie, M. F., Cutri, R. M., Stiening, R., et al. 2006, AJ, 131, 1163

Soderberg, A. M., Chakraborti, S., Pignata, G., et al. 2010, Nature, 463, 513

Sollerman, J., Östlin, G., Fynbo, J. P. U., et al. 2005, New Astron., 11, 103

Solomon, P. M., Downes, D., Radford, S. J. E., \& Barrett, J. W. 1997, ApJ, 478, 144

Speagle, J. S., Steinhardt, C. L., Capak, P. L., \& Silverman, J. D. 2014, ApJS, 214,15

Spring, E. F., \& Michałowski, M. J. 2017, MNRAS, 471, L101

Stanway, E. R., Levan, A. J., Tanvir, N., et al. 2015a, MNRAS, 446, 3911

Stanway, E. R., Levan, A. J., Tanvir, N. R., Wiersema, K., \& van der Laan, T. P. R. 2015b, ApJ, 798, L7

Stott, J. P., Sobral, D., Swinbank, A. M., et al. 2014, MNRAS, 443, 2695

Strauss, M. A., Huchra, J. P., Davis, M., et al. 1992, ApJS, 83, 29

Stritzinger, M., Phillips, M. M., Morrell, N., Salgado, F., \& Folatelli, G. 2009, Cent. Bureau Electron. Tel., 1751

Thöne, C. C., Fynbo, J. P. U., Östlin, G., et al. 2008, ApJ, 676, 1151

Thöne, C. C., Michałowski, M. J., Leloudas, G., et al. 2009, ApJ, 698, 1307

Thöne, C. C., Christensen, L., Prochaska, J. X., et al. 2014, MNRAS, 441 2034

Tully, R. B., Shaya, E. J., Karachentsev, I. D., et al. 2008, ApJ, 676, 184

Vergani, S. D., Salvaterra, R., Japelj, J., et al. 2015, A\&A, 581, A102

Vergani, S. D., Palmerio, J., Salvaterra, R., et al. 2017, A\&A, 599, A120

Weilbacher, P. M., Streicher, O., Urrutia, T. 2014, in Astronomical Data Analysis Software and Systems XXIII, eds. N. Manset, \& P. Forshay, ASP Conf. Ser. 485,451

Wilson, W. E., Ferris, R. H., Axtens, P., et al. 2011, MNRAS, 416, 832
Wright, E. L., Eisenhardt, P. R. M., Mainzer, A. K., et al. 2010, AJ, 140, 1868

Young, J. S., Xie, S., Kenney, J. D. P., \& Rice, W. L. 1989, ApJS, 70, 699

1 Astronomical Observatory Institute, Faculty of Physics, Adam Mickiewicz University, ul. Słoneczna 36, 60-286 Poznań, Poland e-mail: michal.michalowski@amu.edu.pl

2 Scottish Universities Physics Alliance (SUPA), Institute for Astronomy, University of Edinburgh, Royal Observatory, Blakford Hill, EH9 3HJ Edinburgh, UK

3 Sterrenkundig Observatorium, Universiteit Gent, Krijgslaan 281-S9, 9000 Gent, Belgium

${ }^{4}$ Department of Physics and Astrophysics, Vrije Universiteit Brussel, Pleinlaan 2, 1050 Brussels, Belgium

5 European Southern Observatory, Alonso de Córdova 3107, Vitacura, Santiago, Chile

${ }^{6}$ Max-Planck-Institut für Extraterrestrische Physik, Giessenbachstraße, 85748 Garching bei München, Germany

7 Finnish Centre for Astronomy with ESO (FINCA), University of Turku, Väisäläntie 20, 21500 Piikkiö, Finland

8 Tuorla Observatory, Department of Physics and Astronomy, University of Turku, Väisäläntie 20, 21500 Piikkiö, Finland

${ }^{9}$ National Centre for Radio Astrophysics, TIFR, Ganeshkhind, 411007 Pune, India

10 Dark Cosmology Centre, Niels Bohr Institute, University of Copenhagen, Juliane Maries Vej 30, 2100 Copenhagen $\emptyset$, Denmark

11 Visitor Scientist at Department of Physics, Faculty of Science, University of Zagreb, Bijenička cesta 32, 10000 Zagreb, Croatia

12 INAF - Osservatorio Astronomico di Roma, Via di Frascati 33, 00040 Monteporzio Catone, Italy

13 ASI Science Data Centre, Via Galileo Galilei, 00044 Frascati (RM), Italy

14 Hulu LLC., 100084 Beijing, PR China

15 PITT PACC, Department of Physics and Astronomy, University of Pittsburgh, 15260 Pittsburgh, PA, USA

16 INAF - Osservatorio Astrofisico di Arcetri, Largo E. Fermi 5, 50125 Firenze, Italy

17 Centre for Astrophysics Research, University of Hertfordshire, College Lane, AL10 9AB Hatfield, UK

18 Laboratoire AIM-Paris-Saclay, CEA/DSM/Irfu - CNRS - Université Paris Diderot, CE-Saclay, pt courrier 131, 91191 Gif-sur-Yvette, France

19 Thüringer Landessternwarte Tautenburg, Sternwarte 5, 07778 Tautenburg, Germany

20 INAF-OAS Bologna, Via Gobetti 93/3, 40129 Bologna, Italy

21 Technical University of Denmark, Department of Physics, Fysikvej, building 309, 2800 Kgs. Lyngby, Denmark

22 Physics Department, University of Calabria, 87036 Arcavacata di Rende, Italy

23 Instituto de Astrofísica de Andalucía (IAA-CSIC), Glorieta de la Astronomía s/n, 18008 Granada, Spain

${ }^{24}$ Leiden Observatory, Leiden University, PO Box 9513, 2300 RA Leiden, The Netherlands

25 GEPI, Observatoire de Paris, PSL Research University, CNRS, Place Jules Janssen, 92190 Meudon, France 


\section{Appendix A: Long tables and additional figures}

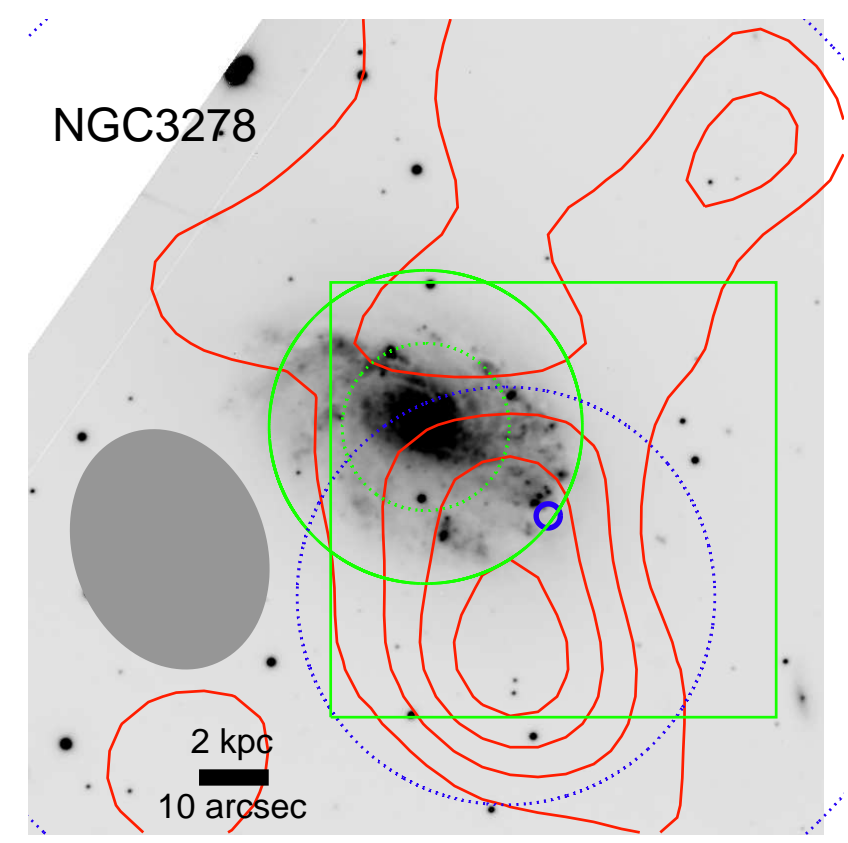

Fig. A.1. Similar to Fig. 3 with additional indication of the spatial coverage of other observations. Green solid and dotted circles correspond to the FWHM of the $\mathrm{CO}(1-0)$ and $\mathrm{CO}(2-1)$ observations from Albrecht et al. (2007). The green square shows the position of the MUSE observations. Blue dotted circles indicate the apertures used to extract the $\mathrm{H}$ I spectrum of the entire galaxy (larger than the image) and the H I peak. 


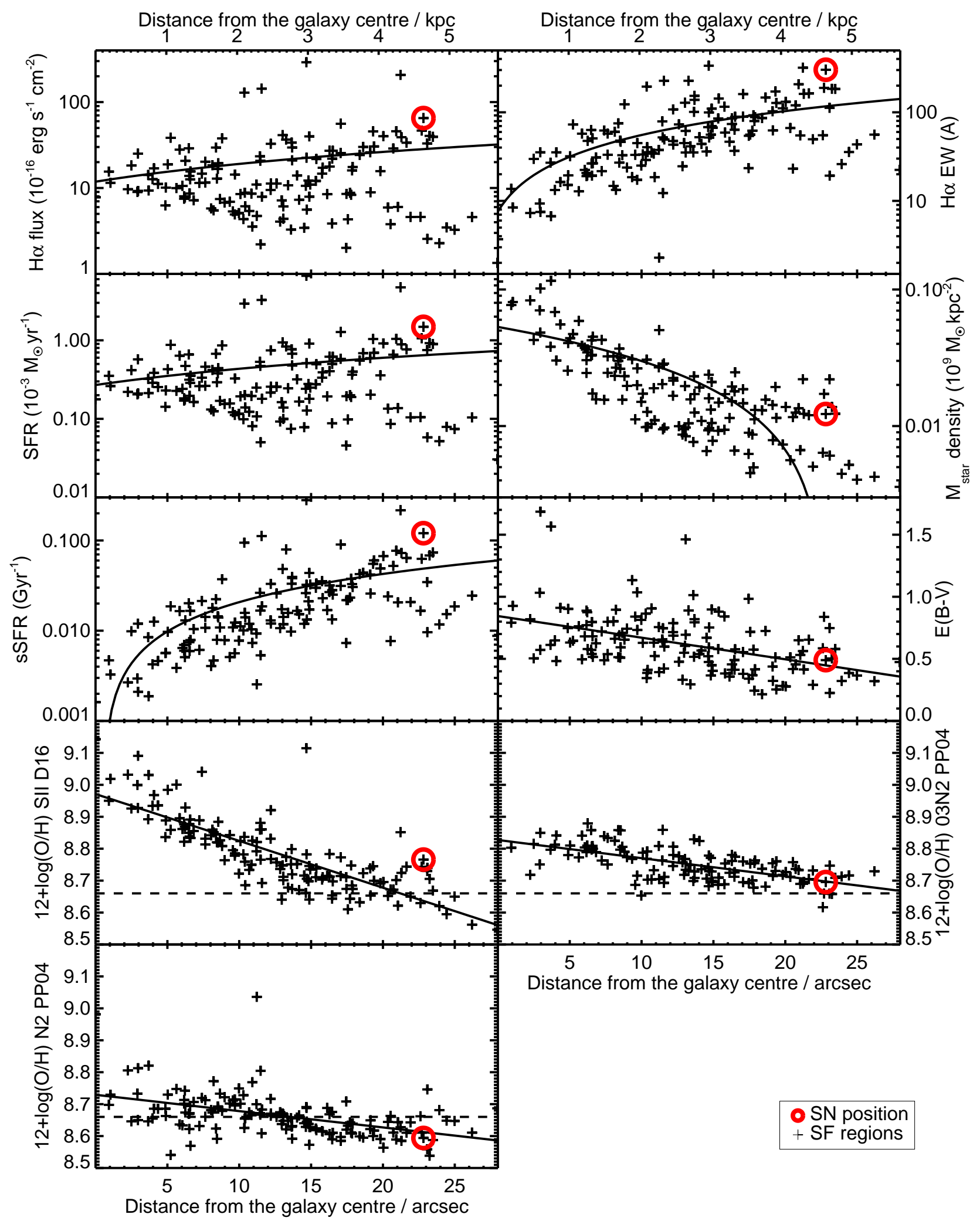

Fig. A.2. Same as Fig. 7 but using measured, not deprojected, distance from the galaxy centre. 
Table A.1. Properties of star-forming regions derived from the MUSE data.

\begin{tabular}{|c|c|c|c|c|c|c|c|c|c|c|c|c|c|c|}
\hline $\begin{array}{c}\text { RA } \\
(\mathrm{deg}) \\
(1) \\
\end{array}$ & $\begin{array}{c}\text { Dec } \\
(\mathrm{deg}) \\
(2) \\
\end{array}$ & $\begin{array}{c}\mathrm{x} \\
\text { (pix) } \\
(3)\end{array}$ & $\begin{array}{c}\mathrm{y} \\
(\mathrm{pix}) \\
(4)\end{array}$ & $\begin{array}{c}\operatorname{dist}_{\text {centre }} \\
\left({ }^{\prime \prime}\right) \\
(5)\end{array}$ & $\begin{array}{c}\text { dist }_{\text {centre }}^{\text {deproj. }} \\
\left({ }^{\prime \prime}\right) \\
(6)\end{array}$ & (7) & $\begin{array}{c}\mathrm{H} \alpha \mathrm{EW} \\
(\AA) \\
(8)\end{array}$ & SFR & $\begin{array}{l}M_{*} \\
(10) \\
\end{array}$ & $\begin{array}{c}\text { sSFR } \\
\left(\mathrm{Gyr}^{-1}\right) \\
(11)\end{array}$ & $\begin{array}{c}E(B-V) \\
(\mathrm{mag}) \\
(12)\end{array}$ & $\begin{array}{l}\frac{12+}{\text { D16 }} \\
(13)\end{array}$ & $\begin{array}{c}03 \mathrm{~N} 2 \\
(14)\end{array}$ & $\begin{array}{c}/ \mathrm{H}) \\
\mathrm{N} 2 \\
(15) \\
\end{array}$ \\
\hline 57.8911250 & -39.9583611 & 156.19 & 148.15 & 22.82 & 23.25 & 65 & 302.3 & 1.484 & 0.012 & 0.120 & & 8.77 & 8.70 & 8.59 \\
\hline & -39.9548683 & 60.98 & 211.01 & & 0.44 & & 3.7 & & & & & 9.14 & & 8.98 \\
\hline & -39.9521484 & 44.13 & 259.97 & & 13.78 & 129 & 195.0 & & 0.031 & & & 8.84 & 8.77 & 8.63 \\
\hline & -39.9537468 & 131.51 & 231.20 & 4.67 & 18.54 & 291 & & & 0.024 & & & .11 & 8.68 & 8.69 \\
\hline & -39.9581451 & 149.19 & 152.03 & 20 & 21.65 & 207 & & & & & & .85 & 69 & 8.63 \\
\hline 57.8973999 & -39.9568672 & 69.61 & 175.03 & 7.40 & 10.22 & 15 & 62.3 & & 0.016 & & & 9.04 & 84 & 8.72 \\
\hline 57.8970337 & -39.9512634 & 74.66 & 275.90 & & & 37 & & & & & & 8.77 & & 8.62 \\
\hline & -39.9519005 & 103.72 & 264.44 & & 20.99 & 21 & 153.4 & & & & & .67 & & 8.59 \\
\hline & -39.952 & 114.24 & & & 18.70 & 41 & & & & & & 8.75 & 8.77 & 8.67 \\
\hline 157.8968048 & -39.9537582 & 77.82 & 231.00 & & 7.99 & 38 & & & & & & 8.86 & & 8.54 \\
\hline & -39.9537697 & 98.66 & 230.79 & & 11.71 & 17 & & & & & & 8.82 & 8.80 & 8.69 \\
\hline & -39.951805 & 43.7 & 266. & & 5 & 143 & 227 & & & & & 86 & & 8.61 \\
\hline & & & & & & & & & & & & 92 & & 8.66 \\
\hline & -39.952434 & & & & 12.73 & 38 & & & & & & 81 & & 8.68 \\
\hline & -39.9531555 & 55.92 & 241.84 & & 8.86 & 21 & & & & & & .93 & 8.88 & 8.67 \\
\hline & -39.953250 & 63.9 & 240.13 & & & 28 & & & & & & 90 & & 8.64 \\
\hline & -39.9 & 91. & & & 15.3 & & & & & & & 72 & & 8.75 \\
\hline & -39.9 & 126. & & & & & & & & & & 8.70 & & 8.62 \\
\hline & -39.95 & 1 & 211.16 & 2 & 3.32 & 46 & & & & & & 8.70 & & 8.59 \\
\hline 157.89 & -39.95 & 1 & & & 4 & & & & & & & 74 & & 8.62 \\
\hline & -39.95 & & & & & & & & & & & 80 & & 8.63 \\
\hline & & & & & & & & & & & & 74 & & 8.60 \\
\hline & & & & & & 34 & & & & & & 8.74 & & 8.61 \\
\hline 8424 & -39.951240 & 63.5 & 276.31 & & & 4 & & & & & & 8.71 & & 8.65 \\
\hline & -39.950836 & & & & 21. & & & & & & & 8.72 & & 8.64 \\
\hline & & & & & & & & & & & & 8.75 & & 8.66 \\
\hline & -39.9 & & & & & 6 & & & & & & 8.71 & 8.73 & 8.65 \\
\hline & & & & & & 2 & & & & & & 8.71 & & 8.71 \\
\hline & -39.951 & & 272.5 & & & & & & & & & 8.65 & 8.73 & 8.59 \\
\hline & -39.95 & 27. & & & & & & & & & & 79 & & 8.70 \\
\hline & -39.9 & 12. & & & & & & & & & & 86 & 8.76 & 8.67 \\
\hline & -39.9 & & & & & & & & & & & 85 & 86 & 8.62 \\
\hline & -39.95 & 44.9 & & & & & & & & & & 8.83 & 8.83 & 8.66 \\
\hline & -39.9536972 & & & & & & & & & & & 8.86 & 8.80 & 8.63 \\
\hline & & & & & & & & & & & & 8.94 & & 8.69 \\
\hline 424 & -39.9 & 63 & 249.12 & & 11.51 & 12 & 33.2 & & & & & 8.81 & 8.75 & 8.71 \\
\hline & -39.9 & & & & & & & & & & & 87 & & 8.74 \\
\hline & -39.9 & & & & & & & & & & & 8.84 & 8.81 & 8.67 \\
\hline & & & & & & 10 & & & & & & 8.89 & 8.78 & 8.66 \\
\hline 5443 & -39.9541626 & 53.8 & 223.72 & & 3.60 & & 9.3 & & 0.070 & & & 9.00 & 8.75 & 8.73 \\
\hline .8975677 & -39.9541245 & 67.29 & 224.40 & & & 9 & 7.5 & & & & & 9.09 & $\ldots$ & 8.81 \\
\hline 157.8958130 & -39.9542313 & 91.5 & 222.48 & & & 14 & 26.4 & & & & & 8.88 & 8.80 & 8.62 \\
\hline & -39.953319 & 107.5 & & & & & & & & & & 8.75 & 8.73 & 8.67 \\
\hline & -39.9538879 & 119.30 & & & & 9 & & & & & & 8.83 & 8.75 & 8.72 \\
\hline 157.8935699 & -39.9532013 & 122.46 & 241.02 & & & 30 & 138.2 & & & & & 8.72 & 8.73 & 8.63 \\
\hline 157.8960419 & -39.9515724 & & 270.34 & & & 6 & & & & & & 8.70 & & 8.67 \\
\hline & -39.9520073 & 114.6 & 262.52 & 14.8 & 22.2 & 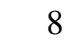 & & & & & & 8.64 & 8.70 & 8.63 \\
\hline & -39.9548950 & & & & & & 27.0 & & & & & 8.89 & 8.80 & 8.65 \\
\hline 157.8983917 & -39.9547997 & 55.93 & 212.25 & 1.04 & 1.14 & 11 & 8.4 & 0.261 & 0.081 & 0.003 & 0.93 & 9.02 & $\ldots$ & 8.73 \\
\hline
\end{tabular}

Notes. The first row is the SN2009bb position and the second is the centre of the galaxy. The last row shows the sum of the individual regions for extensive properties ( $\mathrm{H} \alpha$ flux and SFR) and the average for the intensive properties (equivalent width, extinction, and metallicities). (1) Right Ascension. (2) Declination. (3), (4) MUSE pixel position. (5) Distance from the galaxy centre. (6) Deprojected distance from the galaxy centre (Sect. 2.3). (7) $\mathrm{H} \alpha$ flux in $10^{-16} \mathrm{erg} \mathrm{s}^{-1} \mathrm{~cm}^{-2}$. (8) $\mathrm{H} \alpha$ equivalent width. (9) Star formation rate in $10^{-3} M_{\odot} \mathrm{yr}^{-1}$. (10) Stellar mass in $10^{9} M_{\odot}$. (11) Specific star formation rate (三SFR/ $M_{*}$ ). (12) Extinction. (13) Metallicity based on [SII], [NII], and H $\alpha$ fluxes (Dopita et al. 2016). (14) Metallicity based on [OII], [NII], $\mathrm{H} \alpha$, and $\mathrm{H} \beta$ lines (Pettini \& Pagel 2004). (15) Metallicity based on [NII] and $\mathrm{H} \alpha$ lines (Pettini \& Pagel 2004). 
Table A.1. continued.

\begin{tabular}{|c|c|c|c|c|c|c|c|c|c|c|c|c|c|c|}
\hline $\begin{array}{c}\text { RA } \\
(\mathrm{deg}) \\
(1)\end{array}$ & $\begin{array}{c}\text { Dec } \\
(\mathrm{deg}) \\
(2)\end{array}$ & $\begin{array}{c}x \\
\text { (pix) } \\
(3)\end{array}$ & $\begin{array}{c}\mathrm{y} \\
\text { (pix) } \\
(4)\end{array}$ & $\begin{array}{c}\operatorname{dist}_{\text {centre }} \\
\left({ }^{\prime \prime}\right) \\
(5)\end{array}$ & $\begin{array}{c}\text { dist }_{\text {centre }}^{\text {deproj. }} \\
\left({ }^{\prime \prime}\right) \\
(6)\end{array}$ & $\begin{array}{l}\mathrm{H} \alpha \\
(7)\end{array}$ & $\begin{array}{c}\mathrm{H} \alpha \mathrm{EW} \\
(\AA) \\
(8)\end{array}$ & SFR & (10) & $\begin{array}{c}\text { sSFR } \\
\left(\mathrm{Gyr}^{-1}\right) \\
(11)\end{array}$ & $\begin{array}{c}E(B-V) \\
(\mathrm{mag}) \\
(12)\end{array}$ & $\begin{array}{l}\frac{12+}{\mathrm{D} 16} \\
(13)\end{array}$ & $\begin{array}{c}03 \mathrm{~N} 2 \\
(14)\end{array}$ & $\begin{array}{c}/ \mathrm{H}) \\
\mathrm{N} 2 \\
(15)\end{array}$ \\
\hline 460 & -39.954 & & 220.1 & 1468 & 17.30 & 22 & 99.6 & 511 & 0.016 & & & 8.78 & 8.78 & 8.69 \\
\hline & -39.9553680 & & & & & & & & & & & & & 8.59 \\
\hline & & & & & & & & & & & & & & 8.63 \\
\hline 157.8939667 & -39.9548569 & 116.98 & & 11.20 & 12.50 & & 37.6 & & & & & 3.79 & 8.81 & 8.70 \\
\hline & -39.9544907 & 21.61 & & & & 11 & & & & & & 3.77 & 78 & 8.67 \\
\hline & -39.9560280 & & & & & & & & & & & 3.89 & & 8.69 \\
\hline & & 6 & & & & & & & & & & & & 8.65 \\
\hline & -39.955 & 62.24 & & 2.95 & 4.29 & & & & & & & .93 & & 8.65 \\
\hline & & & & & & & & & & & & & & 8.69 \\
\hline & -39.9549637 & 143.51 & 209.30 & 16.51 & & & & & & & & 3.70 & 8.77 & 8.61 \\
\hline & -39.9552956 & & 203.33 & & & & & & & & & 3.71 & & 8.61 \\
\hline & -39.956 & 5 & & & & & & & & & & & & 8.62 \\
\hline & & & & & & & & & & & & 78 & & 8.67 \\
\hline & & & & & & & & & & & & & & 8.75 \\
\hline & -39.95 & & & & & & & & & & & 3.83 & & 8.57 \\
\hline & & & & 6.49 & & & & & & & & 3.85 & & 8.67 \\
\hline & -39.9 & 74. & & & & & & & & & & & & 8.73 \\
\hline & & & & & & & & & & & & & & 8.69 \\
\hline & & & & & & 21 & & & & & & .88 & & 8.67 \\
\hline & -39.95 & & 218.2 & & & & & & & & & & & 8.60 \\
\hline & & & & & & & & & & & & & & 8.67 \\
\hline & & & & & & & & & & & & 82 & & 8.70 \\
\hline & & & & & & & & & & & & & & 8.73 \\
\hline & & & & & & & & & & & & 3.76 & & 8.65 \\
\hline & -39.95 & & & & & & & & & & & & & 8.66 \\
\hline & & & & & & & & & & & & & & 8.65 \\
\hline & & & & & & & & & & & & .70 & & 8.64 \\
\hline & & & & & & & & & & & & .03 & & 8.81 \\
\hline & & & & & & & & & & & & 95 & & 8.70 \\
\hline & & & & & & & & & & & & & & 8.67 \\
\hline & & & & & & & & & & & & 69 & & 8.68 \\
\hline & & & & & & & & & & & & 80 & & 8.71 \\
\hline & & & & & & & & & & & & 69 & & 8.66 \\
\hline & & & & & & & & & & & & & & 8.61 \\
\hline & & & & & & & & & & & & & & 8.63 \\
\hline & & 5 & & & & & & & & & & 67 & & 8.66 \\
\hline & & & & & & & & & & & & 8.71 & & 8.69 \\
\hline & & & & & & & & & & & & & & 8.61 \\
\hline & & & & & & & & & & & & 57 & & 8.61 \\
\hline & -39.9 & & & & & & & & & & & 72 & 70 & 8.58 \\
\hline & & & & & & & & & & & & 3.71 & 8.66 & 8.54 \\
\hline & & & & & & & & & & & & 3.73 & & 8.56 \\
\hline & -39.95 & & & & & & & & & & & 8.74 & & 8.61 \\
\hline & & & & & & 39 & & & & & & .67 & & 8.59 \\
\hline & -39.9 & & & & & 9 & & & & & & 8.64 & 8.70 & 8.64 \\
\hline & -39.95 & & & & & 40 & & & & & & 8.75 & 8.73 & 8.57 \\
\hline & -39.95 & & & & & & & & & & & 8.77 & 8.69 & 8.58 \\
\hline & & & & & & 16 & & & & & & 8.69 & 8.72 & 8.59 \\
\hline & -39.9582062 & 136.98 & 150.94 & & & 30 & & & & & & 8.70 & 8.76 & 8.62 \\
\hline & -39.9577217 & & & & & 4 & & & & & & 8.78 & $\ldots$ & 8.73 \\
\hline & -39.95 & & & & & & & & & & & 8.75 & 8.68 & 8.61 \\
\hline & -39.9586411 & & & & & 12 & & & & & & 8.71 & 8.69 & 8.62 \\
\hline & -39.9584541 & & & & & & & & & & & 8.64 & 8.72 & 8.64 \\
\hline & -39.9597778 & 113.20 & & 20.53 & & 4 & & & & & & 8.67 & 8.76 & 8.66 \\
\hline & -39.9574585 & & & & & 40 & & & & & & 8.70 & 8.73 & 8.56 \\
\hline & -39.9 & & & & & 9 & & & & & & 8.65 & 8.71 & 8.65 \\
\hline 157.8929443 & -39.9604263 & 131.09 & 110.97 & 24.43 & 28.70 & 3 & 35.7 & 0.079 & 0.005 & 0.015 & 0.39 & 8.59 & 8.72 & 8.65 \\
\hline
\end{tabular}


Table A.1. continued.

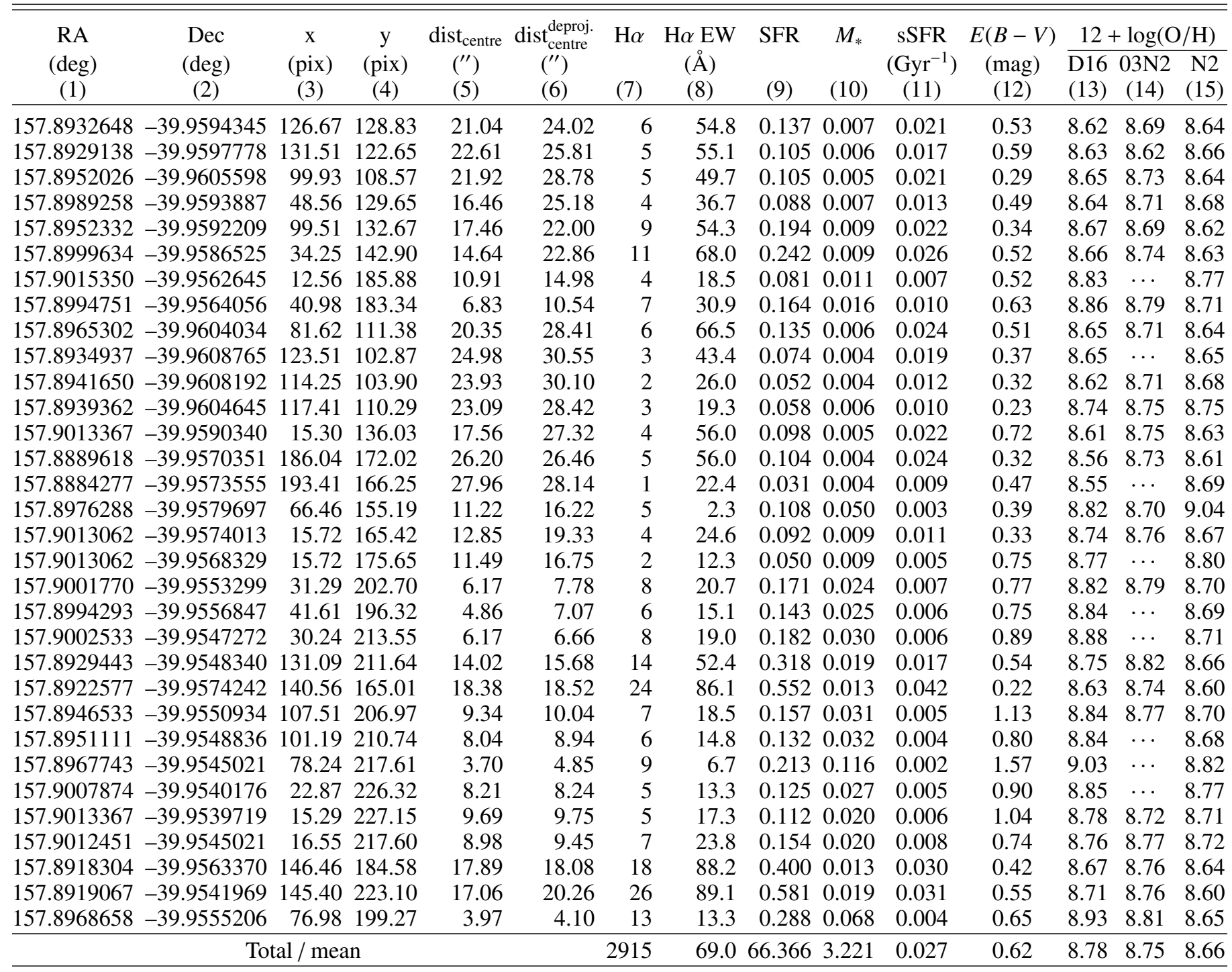

